\title{
EXISTENCE OF SPECTRAL GAPS, COVERING MANIFOLDS AND RESIDUALLY FINITE GROUPS
}

\author{
FERNANDO LLEDÓ AND OLAF POST
}

\begin{abstract}
In this paper we present two construction procedures of covering manifolds $X \rightarrow M$ with residually finite covering transformation group $\Gamma$ such that the spectrum of the Laplacian $\Delta_{X}$ has at least a prescribed number $n$ of spectral gaps, $n \in \mathbb{N}$. If $\Gamma$ has a positive Kadison constant, then we can apply results by Brüning and Sunada to the manifolds constructed here. In this case spec $\Delta_{X}$ has, in addition, band structure and there is an asymptotic estimate for the number $\mathcal{N}(\lambda)$ of components of spec $\Delta_{X}$ that intersect the interval $[0, \lambda]$. Finally, we present several classes of examples of residually finite groups that fit with our construction procedure and study their mutual relations.
\end{abstract}

\section{INTRODUCTION}

The analysis of spectral properties of a Schrödinger operator $H=-\Delta+V$ on $\mathbb{R}^{d}, d \geq 2$, is radically simplified if there is an action of a discrete abelian group $\Gamma_{\mathrm{ab}}=\mathbb{Z}^{d}$ on $\mathbb{R}^{d}$ commuting with $H$. In this case, the potential is completely determined on a (relatively compact) fundamental domain $D$ and one can decompose $H$ as a direct integral over the character group $\widehat{\Gamma}_{\mathrm{ab}}$. If the potential $V$ has high barriers near the boundary $\partial D$, then gaps appear in the spectrum spec $H$. Here, a spectral gap means an interval $(\alpha, \beta)$ with $(\alpha, \beta) \cap \operatorname{spec} H=\emptyset$ and $\alpha, \beta \in \operatorname{spec} H$. For a review on high contrast media see [HP03].

The Laplace operator $\Delta_{X}$ on a Riemannian manifold $(X, g)$ has a formal analogy with the Schödinger operator due to the influence of the non-trivial geometry. Therefore, it is natural to ask whether one can generate some effects of the periodic potential $V$ by purely geometrical means. Specifically, can we generate spectral gaps in spec $\Delta_{X}$ for a covering manifold $X$ with transformation group $\Gamma$ by deforming appropriately the metric $g$ ?

The positive answer to this question is based on the following intuitive idea. The effect of high barriers of the potential on the spectrum is replaced by the following geometrical picture: One decouples in this case neighbouring translates of a the fundamental domain by means of a metric $g_{\varepsilon}$ on $X$, where the junctions between different translates of a fundamental domain are small enough (of order

Date: July 15, 2005.

1991 Mathematics Subject Classification. 58J50, 35P15, 20E26, 57M10.

Key words and phrases. covering manifolds, spectral gaps, residually finite groups, min-max principle. 
$\varepsilon$, see Fig. 1). The structure of the discrete group $\Gamma$ enters crucially when we

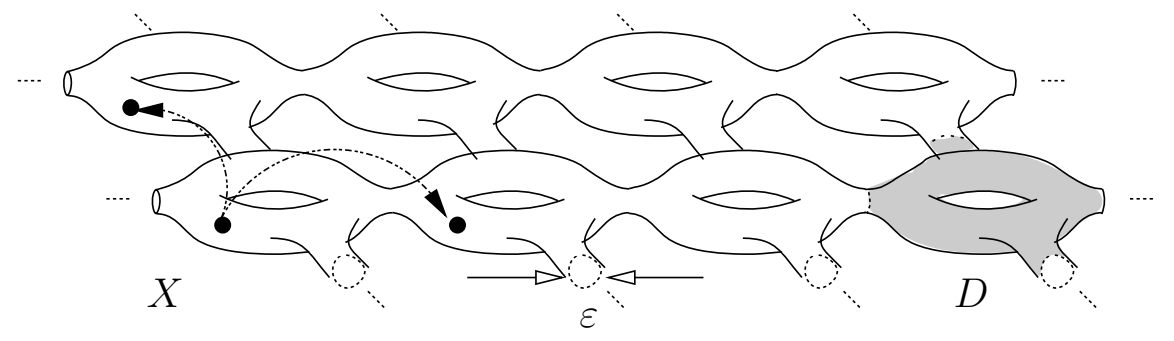

Figure 1. A covering manifold $X$ with fundamental domain $D$. The junctions between different translates of $D$ are of order $\varepsilon$.

implement mathematically the previous idea. In fact, for abelian groups the existence of covering manifolds with a prescribed number $n \in \mathbb{N}$ of spectral gaps was shown in [Pos03] (cf. also the references therein and [BEG03]). The methods presented in the former paper (based on Floquet theory) can be extended to (non-abelian) type I groups (cf. [LP04] and Section 5). The reason for this relies on the following facts: first, the dual $\widehat{\Gamma}$ (i.e., the set of all equivalence classes of continuous irreducible representations) of a type I group is a nice measure space (smooth in the terminology of [Mac76, Ch. 2]) and, second, the fibres in the direct integral decomposition over $\widehat{\Gamma}$ of the right regular representation of $\Gamma$ are finite-dimensional.

In this paper we will present a new method to show the existence of a prescribed number of spectral gaps of $\Delta_{X}$ for covering manifolds $X \rightarrow M$ with residually finite covering transformation groups. This is a much wider class of groups including type I groups, but also free groups or Fuchsian groups. Roughly speaking, a residually finite group has a lot of normal subgroups with finite index. Geometrically, this is equivalent to the fact that one can approximate the covering $X \rightarrow M$ by a sequence of finite coverings $M_{i} \rightarrow M$ (cf. Section 6 for further details); such coverings are also called a tower of coverings. The relation between the spectrum of $\Delta_{X}$ and the spectra of $\Delta_{M_{i}}$ was established in [ASS94, Ada95]. Moreover, if the group $\Gamma$ is amenable, then spec $\Delta_{X}$ is completely determined by $\operatorname{spec} \Delta_{M_{i}}, i \in \mathbb{N}$ (cf. Theorem 6.6).

Under a further assumption, namely, that $\Gamma$ has a positive Kadison constant (cf. Section 7), we can apply results by Sunada and Brüning [Sun92, BS92] to the manifolds $\left(X, g_{\varepsilon}\right)$ constructed here: We obtain in this case, that spec $\Delta_{X}$ has band structure and that there is an asymptotic upper and lower estimate for the number $\mathcal{N}(\lambda)$ of components of the spectrum that intersect the interval $[0, \lambda]$.

To prove the existence of spectral gaps for $\Delta_{X}$, where $\left(X, g_{\varepsilon}\right)$ is the covering manifold mentioned before, one begins by studying the spectral behaviour of the Dirichlet, respectively, Neumann Laplacian $\Delta_{D}$ on the fundamental domain $D$. 
Since their spectrum is purely discrete we can introduce the following intervals

$$
I_{k}(\varepsilon):=\left[\lambda_{k}^{-}(\varepsilon), \lambda_{k}^{+}(\varepsilon)\right]
$$

which are non-empty due to the min-max principle. Here, $\lambda_{k}^{+/-}(\varepsilon)$ denotes the $k$ th Dirichlet, resp., Neumann eigenvalue written in ascending order and counted according to multiplicity. One can show that any of these intervals intersects (non-trivially) spec $\Delta_{X}$ (cf. Theorem 3.3). Moreover, by tuning appropriately the parameter $\varepsilon$ it is possible to show that the union

$$
I(\varepsilon):=\bigcup_{k \in \mathbb{N}} I_{k}(\varepsilon)
$$

is a closed set having at least $n$ gaps, i.e., $n+1$ components as a subset of $[0, \infty)$. In a second step, and using either the fact that $\Gamma$ is type I or residually finite, we can show that

$$
\operatorname{spec} \Delta_{\left(X, g_{\varepsilon}\right)} \subset I(\varepsilon) \text { and } I_{k}(\varepsilon) \cap \operatorname{spec} \Delta_{\left(X, g_{\varepsilon}\right)} \neq \emptyset
$$

for each $k$, hence spec $\Delta_{\left(X, g_{\varepsilon}\right)}$ has at least a finite number of spectral gaps.

Note that the manifolds constructed in this paper are, in a sense, complementary to those considered e.g. by Mazzeo or Friedlander [Fri91, Maz91]. In fact, these authors proved that $\lambda_{k+1}^{-}(D) \leq \lambda_{k}^{+}(D)$ for all $k \in \mathbb{N}$, i.e, that $I_{k} \cap I_{k+1} \neq \emptyset$ for all $k \in \mathbb{N}$ provided $D$ is an open subset of $\mathbb{R}^{n}$ or a Riemannian symmetric space of non-compact type. On such a space, we have a priory no information on the existence of gaps. The authors use the Dirichlet-to-Neumann operator and appropriate test functions to show that the Neumann eigenvalue counting function is strictly greater than the corresponding Dirichlet one.

We consider in the present paper $\Delta_{X}$ as a prototype of an elliptic operator and avoid the use of a potential $V$ in order to analyse only the effect of geometry on spec $\Delta_{X}$. Of course, our methods and results may also be extended to more general periodic structures that have a "reasonable" Neumann Laplacian as a lower bound and satisfy the spectral "localisation" result in Theorem 4.3. For example, one can also study periodic operators like $\Delta_{X}+V$, operators on quantum wave guides, more general periodic elliptic operators or operators on metric graphs (cf. e.g. [EP05] for examples of periodic metric graphs with spectral gaps).

It was already observed by Kobayashi, Ono and Sunada [Sun90, KOS89] that many properties of the spectrum of a periodic Schrödinger operator (e.g. band structure, Bloch's property etc.) generalise to the context of abelian coverings. An exception to this is the existence of $L_{2}$-eigenvalues. Some further interesting results were shown by Brooks [Bro81], e.g. that $\Gamma$ is amenable iff $0 \in \operatorname{spec} \Delta_{X}$. Moreover, in [Bro86] there is a combinatorial criterion whether the second eigenvalue of $\Delta_{M_{i}}$ is bounded from below as $i \rightarrow \infty$, where $M_{i} \rightarrow M$ is a tower of coverings.

The paper is organised as follows: In the following section we set up the problem, present the geometrical context and state some results and conventions that 
will be needed later. In Section 3 we present in detail two construction procedures for covering manifolds, such that the corresponding Laplacian will have a prescribed finite number of spectral gaps. Each method is well adapted to a given initial geometrical context (cf. Remark 3.7 as well as Examples 8.3 and 8.4). In Subsection 3.3 we prove a technical result that assures the existence of spectrum of $\Delta_{X}$ outside the gaps. In Section 4 we review briefly the Floquet theory for non-abelian groups which, roughly speaking, consists of a Fourier transformation of the group part $\ell_{2}(\Gamma)$ of $L_{2}(X) \cong \ell_{2}(\Gamma) \otimes L_{2}(D)$. The Floquet theory is applied in Section 5 for coverings with type I groups. Theorem 5.4 shows the existence of spectral gaps in this case. In Section 6 we study a class of covering manifolds with residually finite groups. That is we approximate the covering $X \rightarrow M$ by a sequence of finite coverings $M_{i} \rightarrow M$, where we apply again the Floquet theory. The existence of a prescribed number of spectral gaps for $\Delta_{X}$ for this class of groups is stated in Theorem 6.8. In Section 7 we study residually finite groups $\Gamma$ that in addition have a positive Kadison constant (e.g., free groups). In this case we give a more complete description of the spectrum outside the gaps. In Section 8 we illustrate the results obtained with some classes of examples and point out their mutual relations. Finally, Subsection 8.3 contains an interesting example of a covering with an amenable, not residually finite group which cannot be treated with our methods. We expect though that in this case one can still generate spectral gaps by the construction presented in Section 3.

\section{Geometrical Preliminaries}

2.1. Covering manifolds and Laplacians. We begin fixing our geometrical context and recalling some results that will be useful later on. We denote by $X$ a non-compact Riemannian manifold of dimension $d \geq 2$ and metric $g$. We also assume the existence of a finitely generated (infinite) discrete group $\Gamma$ of isometries acting properly discontinuously and cocompactly on $X$, i.e., for each $x \in X$ there is a neighbourhood $U$ of $x$ such that the sets $\gamma U$ and $\gamma^{\prime} U$ are disjoint if $\gamma \neq \gamma^{\prime}$ and $M:=X / \Gamma$ is compact. Moreover, the quotient $M$ is a Riemannian manifold which also has dimension $d$ and is locally isometric to $X$. In other words, $\pi: X \longrightarrow M$ is a Riemannian covering space with covering transformation group $\Gamma$. We call such a manifold $\Gamma$-periodic or simply periodic. All groups $\Gamma$ appearing in this paper will satisfy the preceding properties.

We also fix a fundamental domain $D$, i.e., an open set $D \subset X$ such that $\gamma D$ and $\gamma^{\prime} D$ are disjoint for all $\gamma \neq \gamma^{\prime}$ and $\bigcup_{\gamma \in \Gamma} \gamma \bar{D}=X$. We always assume that $\bar{D}$ is compact and that $\partial D$ is piecewise smooth. If not otherwise stated we also assume that $D$ is connected. Note that we can embed $D \subset X$ isometrically into the quotient $M$. In the sequel, we will not always distinguish between $D$ as a subset of $X$ or $M$ since they are isometric. For details we refer to [Rat94, §6.5].

As a prototype for an elliptic operator we consider the Laplacian $\Delta_{X}$ on a Riemannian manifold $(X, g)$ acting on a dense subspace of the Hilbert space 
$L_{2}(X)$ with norm $\|\cdot\|_{X}$. For the formulation of the Theorems 5.4 and 6.8 and at other places, it is useful to denote explicitly the dependence on the metric, since we deform the manifold by changing the metric. In this case we will write $\Delta_{(X, g)}$ for $\Delta_{X}$ or $L_{2}(X, g)$ for $L_{2}(X)$.

The positive self-adjoint operator $\Delta_{X}$ can be defined in terms of a suitable quadratic form $q_{X}$ (see e.g. [Kat95, Chapter VI], [RS80] or [Dav96]). Concretely we have

$$
q_{X}(u):=\|d u\|_{X}^{2}=\int_{X}|d u|^{2} \mathrm{~d} X, \quad u \in C_{\mathrm{c}}^{\infty}(X) .
$$

In coordinates we write the pointwise norm of the 1 -form $d u$ as

$$
|d u|^{2}=\sum_{i, j} g^{i j} \partial_{i} u \partial_{j} \bar{u}
$$

where $\left(g^{i j}\right)$ is the inverse of the metric tensor $\left(g_{i j}\right)$ in a chart. Taking the closure of the quadratic form we can extend $q_{X}$ onto the Sobolev space

$$
\mathcal{H}^{1}(X)=\mathcal{H}^{1}(X, g)=\left\{u \in L_{2}(X) \mid q_{X}(u)<\infty\right\} .
$$

As usual the operator $\Delta_{X}$ is related with the quadratic form by the formula $\left\langle\Delta_{X} u, u\right\rangle=q_{X}(u), u \in C_{\mathrm{c}}^{\infty}(X)$. Since the metric on $X$ is $\Gamma$-invariant, the Laplacian $\Delta_{X}$ (i.e., its resolvent) commutes with the translation on $X$ given by

$$
\left(T_{\gamma} u\right)(x):=u\left(\gamma^{-1} x\right), \quad u \in L_{2}(X), \gamma \in \Gamma .
$$

Operators with this property are called periodic.

For an open, relatively compact subset $D \subset X$ with sufficiently smooth boundary $\partial D$ (e.g. Lipschitz) we define the Dirichlet (respectively, Neumann) Laplacian $\Delta_{D}^{+}$(resp., $\Delta_{D}^{-}$) via its quadratic form $q_{D}^{+}$(resp., $q_{D}^{-}$) associated to the closure of $q_{D}$ on $C_{\mathrm{c}}^{\infty}(D)$, the space of smooth functions with compact support, (resp., $C^{\infty}(\bar{D})$, the space of smooth functions with continuous derivatives up to the boundary). We also use the notation $\mathcal{H}^{1}(D)=\operatorname{dom} q_{D}^{+}\left(\operatorname{resp} ., \mathcal{H}^{1}(D)=\operatorname{dom} q_{D}^{-}\right)$. Note that the usual boundary condition of the Neumann Laplacian occurs only in the operator domain via the Gauß-Green formula. Since $\bar{D}$ is compact, $\Delta_{D}^{+}$ has purely discrete spectrum $\lambda_{k}^{+}, k \in \mathbb{N}$. It is written in ascending order and repeated according to multiplicity. The same is true for the Neumann Laplacian and we denote the corresponding purely discrete spectrum by $\lambda_{k}^{-}, k \in \mathbb{N}$.

One of the advantages of the quadratic form approach is that one can easily read off from the inclusion of domains an order relation for the eigenvalues. In fact, by the the min-max principle we have

$$
\lambda_{k}^{ \pm}=\inf _{L_{k}} \sup _{u \in L_{k} \backslash\{0\}} \frac{q_{D}^{ \pm}(u)}{\|u\|^{2}},
$$

where the infimum is taken over all $k$-dimensional subspaces $L_{k}$ of the corresponding quadratic form domain $\operatorname{dom} q_{D}^{ \pm}$, cf. e.g. [Dav96]. Then the inclusion

$$
\operatorname{dom} q_{D}^{+}=\mathcal{H}^{1}(D) \subset \mathcal{H}^{1}(D)=\operatorname{dom} q_{D}^{-}
$$


implies the following important relation between the corresponding eigenvalues

$$
\lambda_{k}^{+} \geq \lambda_{k}^{-} .
$$

This means, that the Dirichlet $k$-th eigenvalue is in general larger than the $k$-th Neumann eigenvalue and this justifies the choice of the labels + , respectively, - .

\section{Construction of periodic manifolds}

In the present section we will give two different construction procedures (labelled by the letters ' $\mathrm{A}$ ' and 'B') for covering manifolds, such that the corresponding Laplacian will have a prescribed finite number of spectral gaps. In contrast with [Pos03] (where only abelian groups were considered) we will base the construction on the specification of the quotient space $M=X / \Gamma$. By doing this, the spectral convergence result in Theorem 3.1 becomes manifestly independent of the fact whether $\Gamma$ is abelian or not.

Both constructions are done in two steps: first, we specify in two ways the quotient $M$ together with a family of metrics $g_{\varepsilon}$. Second, we construct in either case the covering manifold with covering transformation group $\Gamma$ which has $r$ generators. In the last section we will localise the spectrum of the covering Laplacian in certain intervals given by an associated Dirichlet, respectively, Neumann eigenvalue problem. Some reasons for presenting two different methods (A) and (B) are formulated in a final remark of this section.

3.1. Construction of the quotient. In the following two methods we define a family of Riemannian manifolds $\left(M, g_{\varepsilon}\right)$ that converge to a Riemannian manifold $(N, g)$ of the same dimension (cf. Figure 2). In each case we will also specify a domain $D \subset M$ (in the following section $D$ will become a fundamental domain of the corresponding covering):

(1A) Attaching $r$ handles: We construct the manifold $M$ by attaching $r$ handles diffeomorphic with $C:=(0,1) \times \mathbb{S}^{d-1}$ to a given $d$-dimensional compact orientable manifold $N$ with metric $g$. For simplicity we assume that $N$ has no boundary. Concretely, for each handle we remove two small discs of radius $\varepsilon>0$ from $N$, denote the remaining set by $R_{\varepsilon}$ and identify $\{0\} \times \mathbb{S}^{d-1}$ with the boundary of the first hole and $\{1\} \times \mathbb{S}^{d-1}$ with the boundary of the second hole. We denote by $D$ the open subset of $M$ where the mid section $\{1 / 2\} \times \mathbb{S}^{d-1}$ of each handle is removed.

One can finally define a family of metrics $\left(g_{\varepsilon}\right)_{\varepsilon}, \varepsilon>0$, on $M$ such that the diameter and length of the handle is of order $\varepsilon$ (see e.g. [Pos03, CF81]). In this situation the handles shrink to a point as $\varepsilon \rightarrow 0$. Note that $\left(R_{\varepsilon}, g\right)$ can be embedded isometrically into $(N, g)$, resp., $\left(M, g_{\varepsilon}\right)$. This fact will we useful for proving Theorem 3.3.

(1B) Conformal change of metric: In the second construction, we start with an arbitrary compact $d$-dimensional Riemannian manifold $M$ with metric $g$. We consider only the case $d \geq 3$ (for a discussion of some 
two-dimensional examples see [Pos03]). Moreover, we assume that $N$ and $D$ are two open subsets of $M$ such that (i) $\partial N$ is smooth, (ii) $\bar{N} \subset$ $D$, (iii) $\bar{D}=M$ and (iv) $D \backslash N$ can completely be described by Fermi coordinates (i.e., coordinates $(r, y), r$ being the distance from $N$ and $y \in$ $\partial N)$ up to a set of measure 0 (cf. Figure $2(\mathrm{~B})$ ). The last assumption assures that $N$ is in some sense large in $D$.

Suppose in addition, that $\rho_{\varepsilon}: M \longrightarrow(0,1], \varepsilon>0$, is a family of smooth functions such that $\rho_{\varepsilon}(x)=1$ if $x \in N$ and $\rho_{\varepsilon}(x)=\varepsilon$ if $x \in M \backslash N$ and $\operatorname{dist}(x, \partial N) \geq \varepsilon^{d}$. Then $\rho_{\varepsilon}$ converges pointwise to the characteristic function of $N$. Furthermore, the Riemannian manifold $\left(M, g_{\varepsilon}\right)$ with $g_{\varepsilon}:=$ $\rho_{\varepsilon}^{2} g$ converges to $(N, g)$ in the sense that $M \backslash N$ shrinks to a point in the metric $g_{\varepsilon}$.
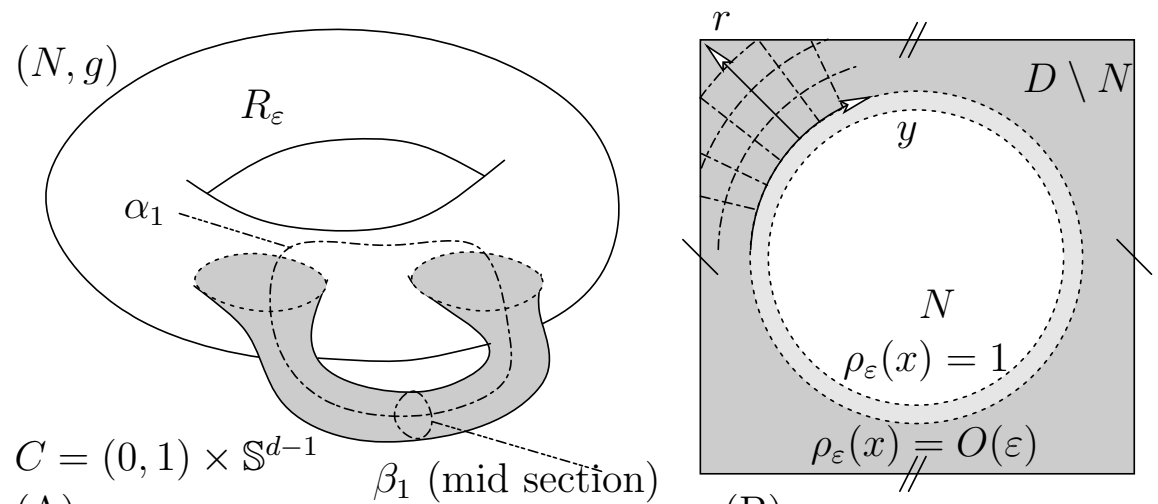

(A)

(B)

FiguRE 2. Two constructions of a family of manifold $\left(M, g_{\varepsilon}\right), \varepsilon>$ 0: In both cases, the grey area has a length scale of order $\varepsilon$ in all directions. (A) We attach $r$ handles (here $r=1$ ) of diameter and length of order $\varepsilon$ to the manifold $(N, g)$. We also denoted the two cycles $\alpha_{1}$ and $\beta_{1}$. (B) We change the metric conformally to $g_{\varepsilon}=\rho_{\varepsilon}^{2} g$. The grey area $D \backslash N$ (with Fermi coordinates in the upper left corner) shrinks conformally to a point as $\varepsilon \rightarrow 0$ whereas $N$ remains fixed. Note that the opposite sides of the square are identified (to obtain a torus as manifold $M$ ).

Now we can formulate the following spectral convergence result which was proven in [Pos03]:

Theorem 3.1. Suppose $\left(M, g_{\varepsilon}\right)$ and $D \subset M$ are constructed as in parts (1A) or (1B) above. In Case (1B) we assume in addition that $d \geq 3$. Then

$$
\lambda_{k}^{ \pm}(\varepsilon) \rightarrow \lambda_{k}(0)
$$


as $\varepsilon \rightarrow 0$ for each $k$. Here, $\lambda_{k}^{ \pm}(\varepsilon)$ denotes the $k$-th Dirichlet, resp., Neumann eigenvalue of the Laplacian on $\left(D, g_{\varepsilon}\right)$ whereas $\lambda_{k}(0)$ is the $k$-th eigenvalue of $(N, g)$ (with Neumann boundary conditions at $\partial N$ in Case (1B)).

3.2. Construction of the covering spaces. Given $\left(M, g_{\varepsilon}\right)$ and $D$ as in the previous subsection, we will associate a Riemannian covering $\pi:\left(X, g_{\varepsilon}\right) \longrightarrow\left(M, g_{\varepsilon}\right)$ with covering transformation group $\Gamma$ such that $D$ is a fundamental domain. Note that we identify $D \subset M$ with a component of the lift $\widetilde{D}:=\pi^{-1}(D)$. Moreover, $\Gamma$ is isomorphic to a normal subgroup of the fundamental group $\pi_{1}(M)$.

(2A) Suppose that $\Gamma$ is a discrete group with $r$ generators $\gamma_{1}, \ldots, \gamma_{r}$. We will construct a $\Gamma$-covering $\left(X, g_{\varepsilon}\right) \rightarrow\left(M, g_{\varepsilon}\right)$ with fundamental domain $D$ where $D$ and $\left(M, g_{\varepsilon}\right)$ are given as in Part (1A) of the previous subsection. Roughly speaking, we glue together $\Gamma$ copies of $D$ along the handles according to the Cayley graph of $\Gamma$ w.r.t. the generators $\gamma_{1}, \ldots, \gamma_{r}$. For convenience of the reader, we specify the construction:

The fundamental group of $M$ is given by $\pi_{1}(M)=\pi_{1}(N) * \mathbb{Z}^{* r}$ in the case $d \geq 3$. Here, $G_{1} * G_{2}$ denotes the free product of $G_{1}$ and $G_{2}$, and $\mathbb{Z}^{* r}$ is the free group in $r$ generators $\alpha_{1}, \ldots, \alpha_{r}$. If $d=2$ we know from the classification result for 2-dimensional orientable manifolds that $N$ is diffeomorphic to an $s$-holed torus. In this case the fundamental group is given by

$$
\pi_{1}(M)=\left\langle\alpha_{1}, \beta_{1}, \ldots, \alpha_{r+s}, \beta_{r+s} \mid\left[\alpha_{1}, \beta_{1}\right] \cdot \ldots \cdot\left[\alpha_{r+s}, \beta_{r+s}\right]=e\right\rangle,
$$

where $[\alpha, \beta]:=\alpha \beta \alpha^{-1} \beta^{-1}$ is the usual commutator. We may assume that $\alpha_{i}$ represents the homotopy class of the cycle transversal to the section of the $i$-th handle and that $\beta_{i}$ represents the section itself $(i=1, \ldots, r)$ (cf. Figure $2(\mathrm{~A})$ ).

One easily sees that there exists an epimorphism $\varphi: \pi_{1}(M) \longrightarrow \Gamma$ which maps $\alpha_{i} \in \pi_{1}(M)$ to $\gamma_{i} \in \Gamma(i=1, \ldots, r)$ and all other generators to the unit element $e \in \Gamma$. Note that this map is also well-defined in the case $d=2$, since the relation in (3.1) is trivially satisfied in the case when the $\beta_{i}$ 's are mapped to $e$.

Finally, $\Gamma \cong \pi_{1}(M) / \operatorname{ker} \varphi$, and $X \rightarrow M$ is the associated covering with respect to the universal covering $\widetilde{M} \rightarrow M$ (considered as a principal bundle with discrete fibre $\Gamma$ ) and the natural action of $\Gamma$ on $\pi_{1}(M)$.

Then $X \rightarrow M$ is a normal $\Gamma$-covering with fundamental domain $D$ constructed as in (1A) of the preceding subsection. Here we use the fact that $\alpha_{i}$ is transversal to the section of the handle in dimension 2 .

(2B) Suppose $(X, g) \rightarrow(M, g)$ is a Riemannian covering with fundamental domain $D$ such that $\partial D$ is piecewise smooth. Then $\bar{D}=M$, where we have embedded $D$ into the quotient, cf. [Rat94, Theorem 6.5.8]. According to $(1 \mathrm{~B})$ we can conformally change the metric on $M$, to produce a new covering $\left(X, g_{\varepsilon}\right) \rightarrow\left(M, g_{\varepsilon}\right)$ that satisfies the required properties. 
In both cases, we lift for each $\varepsilon>0$ the metric $g_{\varepsilon}$ from $M$ to $X$ and obtain a Riemannian covering $\left(X, g_{\varepsilon}\right) \rightarrow\left(M, g_{\varepsilon}\right)$. Note that the set $D$ specified in the first step of the previous construction becomes a fundamental domain after the specification of the covering in the second step.

The following statement is a direct consequence of the spectral convergence result in Theorem 3.1:

Theorem 3.2. Suppose $\left(X, g_{\varepsilon}\right) \rightarrow\left(M, g_{\varepsilon}\right)(\varepsilon>0)$ is a family of Riemannian coverings with fundamental domain $D$ constructed as in the previous parts (2A) or (2B). Then for each $n \in \mathbb{N}$ there exists $\varepsilon=\varepsilon_{n}>0$ such that

$$
I(\varepsilon):=\bigcup_{k \in \mathbb{N}} I_{k}(\varepsilon), \quad \text { with } \quad I_{k}(\varepsilon):=\left[\lambda_{k}^{-}(\varepsilon), \lambda_{k}^{+}(\varepsilon)\right]
$$

is a closed set having at least $n$ gaps, i.e., $n+1$ components as subset of $[0, \infty)$. Here, $\lambda_{k}^{ \pm}(\varepsilon)$ denotes the $k$-th Dirichlet, resp., Neumann eigenvalue of the Laplacian on $\left(D, g_{\varepsilon}\right)$.

Proof. First, note that $\left\{\lambda_{k}^{ \pm}(\varepsilon) \mid k \in \mathbb{N}\right\}, \varepsilon \geq 0$, has no finite accumulation point, since the spectrum is discrete. Second, Theorem 3.1 shows that the intervals $I_{k}(\varepsilon)$ reduce to the point $\left\{\lambda_{k}(0)\right\}$ as $\varepsilon \rightarrow 0$. Therefore, $I(\varepsilon)$ is a locally finite union of compact intervals, hence closed.

3.3. Existence of spectrum outside the gaps. In the following subsection we will assure that each Neumann-Dirichlet interval $I_{k}(\varepsilon)$ contains at least one point of spec $\Delta_{\left(X, g_{\varepsilon}\right)}$ provided $\varepsilon$ is small enough. In our general setting described below (cf. Theorems 5.4 and 6.8) we will show the inclusion

$$
\operatorname{spec} \Delta_{\left(X, g_{\varepsilon}\right)} \subset \bigcup_{k \in \mathbb{N}} I_{k}(\varepsilon) \text {. }
$$

It is a priori not clear that each $I_{k}(\varepsilon)$ intersects the spectrum of the Laplacian on $\left(X, g_{\varepsilon}\right)$, i.e., that gaps in $\bigcup_{k \in \mathbb{N}} I_{k}(\varepsilon)$ are also gaps in $\operatorname{spec} \Delta_{\left(X, g_{\varepsilon}\right)}$. If the covering group is amenable, the $k$-th eigenvalue of the Laplacian on the quotient $\left(M, g_{\varepsilon}\right)$ is always an element of $I_{k}(\varepsilon) \cap \operatorname{spec}\left(\Delta_{X}, g_{\varepsilon}\right)$ (cf. the argument in the proof of Theorem 5.4). In general, this need not to be true. Therefore, we need the following theorem which will be used in Theorems 6.8 and 7.3:

Theorem 3.3. With the notation of the previous theorem, we have

$$
I_{k}(\varepsilon) \cap \operatorname{spec} \Delta_{\left(X, g_{\varepsilon}\right)} \neq \emptyset
$$

for all $k \in \mathbb{N}$.

We begin with a general criterion which will be useful to detect points in the spectra of a parameter-dependent family of operators using only its sesquilinear form. A similar result is also stated in [KK03, Lemma 5.1].

Suppose that $H_{\varepsilon}$ is a self-adjoint, non-negative, unbounded operator in a Hilbert space $\mathcal{H}_{\varepsilon}$ for each $\varepsilon>0$. Denote by $\mathcal{H}_{\varepsilon}^{1}:=\operatorname{dom} h_{\varepsilon}$ the Hilbert space 
of the corresponding quadratic form $h_{\varepsilon}$ associated to $H_{\varepsilon}$ with norm $\|u\|_{1}:=$ $\left(h_{\varepsilon}(u)+\|u\|_{\mathcal{H}_{\varepsilon}}\right)^{1 / 2}$ and by $\mathcal{H}_{\varepsilon}^{-1}$ the dual of $\mathcal{H}_{\varepsilon}^{1}$. Note that $H_{\varepsilon}: \mathcal{H}_{\varepsilon}^{1} \longrightarrow \mathcal{H}_{\varepsilon}^{-1}$ is continuous. In the next lemma we characterise for each $\varepsilon$ certain spectral points of $H_{\varepsilon}$.

Lemma 3.4. Suppose there exist a family $\left(u_{\varepsilon}\right) \subset \mathcal{H}_{\varepsilon}^{1}$ and constants $\lambda \geq 0, c>0$ such that

$$
\left\|\left(H_{\varepsilon}-\lambda\right) u_{\varepsilon}\right\|_{-1} \rightarrow 0 \quad \text { as } \quad \varepsilon \rightarrow 0
$$

and $\left\|u_{\varepsilon}\right\| \geq c>0$ for all $\varepsilon>0$, then there exists $\delta=\delta(\varepsilon) \rightarrow 0$ as $\varepsilon \rightarrow 0$ such that

$$
\lambda+\delta(\varepsilon) \in \operatorname{spec} H_{\varepsilon} .
$$

Proof. Suppose that the conclusion is false. Then there exist a sequence $\varepsilon_{n} \rightarrow 0$ and a constant $\delta_{0}>0$ such that

$$
I_{\lambda} \cap \operatorname{spec} H_{\varepsilon_{n}}=\emptyset \quad \text { with } \quad I_{\lambda}:=\left(\lambda-\delta_{0}, \lambda+\delta_{0}\right)
$$

for all $n \in \mathbb{N}$. Denote by $E_{t}$ the spectral resolution of $H_{\varepsilon}$. Then

$$
\begin{aligned}
\left\|\left(H_{\varepsilon}-\lambda\right) u_{\varepsilon}\right\|_{-1}^{2}=\int_{\mathbb{R}_{+} \backslash I_{\lambda}} \frac{(t-\lambda)^{2}}{(t+1)} \mathrm{d}\left\langle E_{t} u_{\varepsilon}, u_{\varepsilon}\right\rangle & \\
\geq & \frac{\delta_{0}^{2}}{\lambda+\delta_{0}+1} \int_{\mathbb{R}_{+} \backslash I_{\lambda}} \mathrm{d}\left\langle E_{t} u_{\varepsilon}, u_{\varepsilon}\right\rangle \geq \frac{c \delta_{0}^{2}}{\lambda+\delta_{0}+1}
\end{aligned}
$$

since $I_{\lambda}$ does not lie in the support of the spectral measure. But this inequality contradicts (3.5).

Remark 3.5. Eq. (3.5) is equivalent to the inequality

$$
\left|h_{\varepsilon}\left(u_{\varepsilon}, v_{\varepsilon}\right)-\lambda\left\langle u_{\varepsilon}, v_{\varepsilon}\right\rangle\right| \leq o(1)\left\|v_{\varepsilon}\right\|_{1} \quad \text { for all } v_{\varepsilon} \in \mathcal{H}_{\varepsilon}^{1}
$$

as $\varepsilon \rightarrow 0$. Note that $o(1)$ could depend on $u_{\varepsilon}$. The advantage of the criterion in the previous lemma is that one only needs to find a family $\left(u_{\varepsilon}\right)$ in the domain of the quadratic form $h_{\varepsilon}$.

We will need the following lemma in order to define a cut-off function with convergent $L_{2}$-integral of its derivative. Its proof is straightforward.

Lemma 3.6. Denote by $h(r):=r^{-d+2}$ if $d \geq 3$ and $h(r)=\ln r$ if $d=2$. For $\varepsilon \in(0,1)$ define

$$
\chi_{\varepsilon}(r):= \begin{cases}0, & 0<r \leq \varepsilon \\ \frac{h(r)-h(\varepsilon)}{h(\sqrt{\varepsilon})-h(\varepsilon)}, & \varepsilon \leq r \leq \sqrt{\varepsilon} \\ 1, & \sqrt{\varepsilon} \leq r\end{cases}
$$

then $\chi_{\varepsilon} \in \mathcal{H}^{1}((0,1))$ and

$$
\left\|\chi_{\varepsilon}^{\prime}\right\|^{2}:=\int_{0}^{1}\left|\chi_{\varepsilon}^{\prime}(r)\right|^{2} r^{d-1} \mathrm{~d} r=o(1) .
$$


Remember that $(N, g)$ is the unperturbed manifold as in Fig. 2. In Case A of Subsection 3.1, we denoted by $R_{\varepsilon}$ the manifold $N$ with a closed ball of radius $\varepsilon$ removed around each point where the handles have been attached (note that $R_{\varepsilon}$ is also contained in $D)$ and denote by $(r, y)$ the polar coordinates around such a point $\left(r=\varepsilon\right.$ corresponds to a component of $\left.\partial R_{\varepsilon}\right)$.

Proof of Theorem 3.3. Let $\varphi$ be the $k$-th eigenfunction of the limit operator $\Delta_{N}$ with eigenvalue $\lambda=\lambda_{k}(0)$. We will treat Case A and B of Subsection 3.1 separately.

(3A) Set $u_{\varepsilon}(r, y):=\chi_{\varepsilon}(r) \varphi(r, y)$ in the polar coordinates described above and $u_{\varepsilon}:=\varphi$ on $R_{\sqrt{\varepsilon}}$. Now, $\|\varphi\|_{R_{\sqrt{\varepsilon}}}^{2} \geq c$ since $\|\varphi\|_{R_{\sqrt{\varepsilon}}}^{2} \rightarrow\|\varphi\|_{N}^{2}>0$ as $\varepsilon \rightarrow 0$. In addition, $u_{\varepsilon} \in \mathcal{H}^{1}\left(R_{\varepsilon}\right) \subset \mathcal{H}^{1}\left(X, g_{\varepsilon}\right)$ and

$$
\begin{aligned}
\mid\left\langle d u_{\varepsilon}, d v_{\varepsilon}\right\rangle & -\lambda\left\langle u_{\varepsilon}, v_{\varepsilon}\right\rangle \mid \\
& =\left|\int_{R_{\varepsilon}}\left[\left\langle d \varphi, d\left(\chi_{\varepsilon} v_{\varepsilon}\right)\right\rangle-\lambda \varphi \overline{\chi_{\varepsilon} v}\right]+\int_{R_{\varepsilon}} \varphi\left\langle d \chi_{\varepsilon}, d v_{\varepsilon}\right\rangle-\int_{R_{\varepsilon}} \bar{v}\left\langle d \varphi, d \chi_{\varepsilon}\right\rangle\right|
\end{aligned}
$$

for all $v_{\varepsilon} \in \mathcal{H}^{1}\left(D_{\varepsilon}\right)$. Now the first integral vanishes since $\varphi$ is the eigenfunction with eigenvalue $\lambda$ on $N$. Note that $\chi_{\varepsilon} v \in \mathcal{H}^{1}\left(R_{\varepsilon}\right)$ can be interpreted as function in $\mathcal{H}^{1}(N)$. The second and third integral can be estimated from above by

$$
\sup _{x \in N}[|\varphi(x)|+|d \varphi(x)|]\left\|\chi_{\varepsilon}^{\prime}\right\|\left\|v_{\varepsilon}\right\|_{1}=o(1)\left\|v_{\varepsilon}\right\|_{1}
$$

since $\varphi$ is a smooth function on an $\varepsilon$-independent space and due to Lemma 3.6. (3B) Set $u_{\varepsilon}:=\varphi$ on $N$ and $u_{\varepsilon}(r, y):=\widetilde{\chi}_{\varepsilon}(r) \varphi(0, y), r>0$, i.e., on $D \backslash N$ with $\tilde{\chi}_{\varepsilon}(r):=\chi_{\varepsilon}\left(\sqrt{\varepsilon}+\varepsilon^{d}-r\right)$, where $\chi_{\varepsilon}$ is defined in (3.7) with $d=2$. Note that $\widetilde{\chi}_{\varepsilon}^{\prime}(r) \neq 0$ only for those $r=\operatorname{dist}(x, \partial N)$ where the conformal factor $\rho_{\varepsilon}(x)=\varepsilon$. Now, $u_{\varepsilon} \in \mathcal{H}^{1}\left(D, g_{\varepsilon}\right) \subset \mathcal{H}^{1}\left(X, g_{\varepsilon}\right)$. Furthermore, for $v_{\varepsilon} \in \mathcal{H}^{1}\left(D, g_{\varepsilon}\right)$ we have

$$
\begin{aligned}
&\left|\left\langle d u_{\varepsilon}, d v_{\varepsilon}\right\rangle-\lambda\left\langle u_{\varepsilon}, v_{\varepsilon}\right\rangle\right| \leq \int_{D \backslash N}\left[\left|\widetilde{\chi}_{\varepsilon}^{\prime}(r) \varphi(0, y) \partial_{r} v_{\varepsilon}\right| \rho_{\varepsilon}^{d-2}\right. \\
&\left.+\left|\widetilde{\chi}_{\varepsilon}(r)\left\langle d_{y} \varphi(0, y), d_{y} v_{\varepsilon}\right\rangle\right| \rho_{\varepsilon}^{d-2}+\lambda \widetilde{\chi}_{\varepsilon}(r)\left|\varphi(0, y) v_{\varepsilon}\right| \rho_{\varepsilon}^{d}\right] \mathrm{d} r \mathrm{~d} y \\
& \leq C\left[\left(\int_{\varepsilon^{d}}^{\sqrt{\varepsilon}+\varepsilon^{d}-\varepsilon}\left|\widetilde{\chi}_{\varepsilon}^{\prime}(r)\right|^{2} \varepsilon^{d-2} \mathrm{~d} r\right)^{\frac{1}{2}}\right. \\
&\left.+\left(\int_{0}^{\sqrt{\varepsilon}}\left|\widetilde{\chi}_{\varepsilon}(r)\right|^{2} \rho_{\varepsilon}^{d-2} \mathrm{~d} r\right)^{\frac{1}{2}}+\left(\int_{0}^{\sqrt{\varepsilon}}\left|\widetilde{\chi}_{\varepsilon}(r)\right|^{2} \rho_{\varepsilon}^{d} \mathrm{~d} r\right)^{\frac{1}{2}}\right]\left\|v_{\varepsilon}\right\|_{1}
\end{aligned}
$$

where we have used that $\varphi$ is the Neumann eigenfunction on $N$. Furthermore, $C$ depends on the supremum of $\varphi$ and $d \varphi$ and on $\lambda$. Note that the conformal factor $\rho_{\varepsilon}$ equals $\varepsilon$ on the support of $\widetilde{\chi}_{\varepsilon}^{\prime}$, therefore, he first integral converges to 0 
since $d \geq 3$. Finally, estimating $\widetilde{\chi}_{\varepsilon}$ and $\rho_{\varepsilon}$ by 1 , the second and third integral are bounded by $\varepsilon^{1 / 4}$.

Remark 3.7. In the present section we have specified two construction procedures for covering manifolds, such that the corresponding Laplacians will have at least a prescribed number of spectral gaps (cf. Sections 5 and 6). The construction specified in method (A) is feasible for every given covering transformation group $\Gamma$ with $r$ generators. Note that this method produces fundamental domains that have smooth boundaries (see e.g. Example 8.3 below).

The construction in (B) applies for every given Riemannian covering $(X, g) \rightarrow$ $(M, g)$, since, by the procedure described, one can modify conformally this covering in order to satisfy the spectral convergence result of Theorem 3.1 (cf. Example 8.4).

\section{Floquet TheORY FOR NON-ABELIAN GROUPS}

The aim of the present section is to state a spectral inclusion result (cf. Theorem 4.3) and the direct integral decomposition of $\Delta_{X}$ (cf. Theorem 4.5) for certain non-abelian discrete groups $\Gamma$. These results will be used to prove the existence of spectral gaps in the situations analysed in the next two sections. A more detailed presentation of the results in this section may be found in [LP04].

4.1. Equivariant Laplacians. We will introduce next a new operator that lies "between" the Dirichlet and Neumann Laplacians and that will play an important role in the following. Suppose $\rho$ is a unitary representation of the discrete group $\Gamma$ on the Hilbert space $H$, i.e., $\rho: \Gamma \longrightarrow \mathcal{U}(H)$ is a homomorphism. We fix a fundamental domain $D$ for the $\Gamma$-covering $X \rightarrow M$.

We now introduce the following space of smooth $\rho$-equivariant functions

$$
C_{\rho}^{\infty}(D, H):=\left\{h \uparrow_{D} \mid h \in C^{\infty}(X, H), \quad h(\gamma x)=\rho_{\gamma} h(x), \quad \gamma \in \Gamma, x \in X\right\} .
$$

This definition coincides with the usual one for abelian groups, cf. [LP04]. Note that we need vector-valued functions $h: X \longrightarrow H$ since the representation $\rho$ acts on the Hilbert space $H$, which, in general, has dimension $\geq 2$.

We define next the so-called equivariant Laplacian (w.r.t. the representation $\rho)$ on $L_{2}(D, H) \cong L_{2}(D) \otimes H$ : Let a quadratic form be defined by

$$
\|d h\|_{D}^{2}:=\int_{D}\|d h(x)\|_{H}^{2} \mathrm{~d} X(x)
$$

for $h \in C_{\rho}^{\infty}(D, H)$, where the integrand is locally given by

$$
\|d h(x)\|_{H}^{2}=\sum_{i, j} g^{i j}(x)\left\langle\partial_{i} h(x), \partial_{j} h(x)\right\rangle_{H}, \quad x \in D .
$$

This generalises Eq. (2.1) to the case of vector-valued functions. We denote the closure by $q_{D}^{\rho}$ and its domain by $\mathcal{H}_{\rho}^{1}(D, H)$. The corresponding non-negative, 
self-adjoint operator on $L_{2}(D, H)$, the $\rho$-equivariant Laplacian, will be denoted by $\Delta_{D, H}^{\rho}($ cf. [Kat95, Chapter VI]).

4.2. Dirichlet-Neumann bracketing. We study in this section the spectrum of a $\rho$-equivariant Laplacian $\Delta^{\rho}$ associated with a finite-dimensional representation $\rho$. In particular, we show that spec $\Delta^{\rho}$ is contained in a suitable set determined by the spectrum of the Dirichlet and Neumann Laplacians on $D$. The key ingredient in dealing with non-abelian groups is the observation that this set is independent of $\rho$.

We begin with the definition of certain operators acting in $L_{2}(D, H)$ and its eigenvalues. We denote by $\lambda_{m}^{-}(H), \lambda_{m}^{\rho}(H)$, resp., $\lambda_{m}^{+}(H)$ the $m$-th eigenvalue of the operator $\Delta_{D, H}^{-}, \Delta_{D, H}^{\rho}$, resp., $\Delta_{D, H}^{+}$corresponding to the quadratic form (4.2) on $\mathcal{H}^{1}(D, H), \mathcal{H}_{\rho}^{1}(D, H)$, resp., $\mathcal{H}^{1}(D, H)$. Recall that $\mathcal{H}^{1}(D, H)$ is the $\mathcal{H}^{1}$ closure of the space of smooth functions $h: D \longrightarrow H$ with support away from $\partial D$ and $\mathcal{H}^{1}(D, H)$ is the closure of the space of smooth functions with derivatives continuous up to the boundary.

The proof of the following lemma follows, as in the abelian case (cf. Eqs. (2.4) and (2.5)), from the reverse inclusions of the quadratic form domains

$$
\mathcal{H}^{1}(D, H) \supset \mathcal{H}_{\rho}^{1}(D, H) \supset \mathcal{H}^{1}(D, H)
$$

and the min-max principle (2.3).

Lemma 4.1. We have

$$
\lambda_{m}^{-}(H) \leq \lambda_{m}^{\rho}(H) \leq \lambda_{m}^{+}(H)
$$

for all $m \in \mathbb{N}$.

From the definition of the quadratic form in the Dirichlet, resp., Neumann case we have that the corresponding vector-valued Laplacians are a direct sum of the scalar operators. Therefore the eigenvalues of the corresponding vector-valued Laplace operators consist of repeated eigenvalues of the scalar Laplacian. We can arrange the former in the following way:

Lemma 4.2. If $n:=\operatorname{dim} H<\infty$ then

$$
\lambda_{m}^{ \pm}(H)=\lambda_{k}^{ \pm}, \quad m=(k-1) n+1, \ldots, k n,
$$

where $\lambda_{k}^{ \pm}$denotes the (scalar) $k$-th Dirichet/Neumann eigenvalue on D.

Proof. Note that $\Delta_{D, H}^{ \pm}$is unitarily equivalent to an $n$-fold direct sum of the scalar operator $\Delta_{D}^{ \pm}$on $L_{2}(D)$ since there is no coupling between the components on the boundary.

Recall the definition of the intervals $I_{k}:=\left[\lambda_{k}^{-}, \lambda_{k}^{+}\right]$in Eq. (3.2) (for simplicity, we omit in the following the index $\varepsilon$ ). From the preceding two lemmas we may collect the $n$ eigenvalues of $\Delta_{D, H}^{\rho}$ which lie in $I_{k}$ :

$$
B_{k}(\rho):=\left\{\lambda_{m}^{\rho}(H) \mid m=(k-1) n+1, \ldots, k n\right\} \subset I_{k}, \quad n:=\operatorname{dim} H .
$$


Therefore, we obtain the following spectral inclusion for equivariant Laplacians. This result will be applied in Theorems 5.4 and 6.8 below.

Theorem 4.3. If $\rho$ is a unitary representation on a finite-dimensional Hilbert space $H$ then

$$
\operatorname{spec} \Delta_{D, H}^{\rho}=\bigcup_{k \in \mathbb{N}} B_{k}(\rho) \subseteq \bigcup_{k \in \mathbb{N}} I_{k}
$$

where $\Delta_{D, H}^{\rho}$ denotes the $\rho$-equivariant Laplacian.

4.3. Non-abelian Floquet transformation. Consider first the right, respectively, left regular representation $R$, resp., $L$ on the Hilbert space $\ell_{2}(\Gamma)$ :

$$
\left(R_{\gamma} a\right)_{\widetilde{\gamma}}=a_{\widetilde{\gamma} \gamma}, \quad\left(L_{\gamma} a\right)_{\widetilde{\gamma}}=a_{\gamma^{-1}}, \quad a=\left(a_{\gamma}\right)_{\gamma} \in \ell_{2}(\Gamma), \quad \gamma, \widetilde{\gamma} \in \Gamma .
$$

Using standard results we introduce the following unitary map (see e.g., [LP04, Section 3 and the appendix] and references cited therein)

$$
F: \ell_{2}(\Gamma) \longrightarrow \int_{Z}^{\oplus} H(z) \mathrm{d} z
$$

for a suitable measure space $(Z, \mathrm{~d} z)$. The map $F$ is a generalisation of the Fourier transformation in the abelian case. Moreover, it transforms the right regular representation $R$ into the following direct integral representation

$$
\widehat{R}_{\gamma}=F R_{\gamma} F^{*}=\int_{Z}^{\oplus} R_{\gamma}(z) \mathrm{d} z, \quad \gamma \in \Gamma .
$$

Remark 4.4. Let $\mathcal{R}$ be the von Neumann algebra generated by all unitaries $R_{\gamma}$, $\gamma \in \Gamma$, i.e.,

$$
\mathcal{R}=\left\{R_{\gamma} \mid \gamma \in \Gamma\right\}^{\prime \prime}
$$

where $\mathcal{R}^{\prime}$ denotes the commutant of $\mathcal{R}$ in $\mathcal{L}\left(\ell_{2}(\Gamma)\right)$. Then we decompose $\mathcal{R}$ with respect to a maximal abelian von Neumann subalgebra $\mathcal{A} \subset \mathcal{R}^{\prime}$ (for a concrete example see Example 4.6). The space $Z$ is the compact Hausdorff space associated, by Gelfand's isomorphism, to a separable $C^{*}$-algebra $\mathcal{C}$, which is strongly dense in $\mathcal{A}$. Furthermore, $\mathrm{d} z$ is a regular Borel measure on $Z$. We may identify the algebra $\mathcal{A}$ with $L_{\infty}(Z, \mathrm{~d} z)$ and since it is maximal abelian, the fibre representations $R(z)$ are irreducible a.e. (see [Wal92, Section $14.8 \mathrm{ff.}$.]).

The generalised Fourier transformation introduced in Eq. (4.6) can be used to decompose $L_{2}(X)$ into a direct integral. In particular, we define for a.e. $z \in Z$ :

$$
(U u)(z)(x):=\sum_{\gamma \in \Gamma} u(\gamma x) R_{\gamma^{-1}}(z) v(z)
$$

where $v:=F \delta_{e} \in \ell_{2}(\Gamma), u \in C_{\mathrm{c}}^{\infty}(X)$ and $x \in D$. The map $U$ extends to a unitary map

$$
U: L_{2}(X) \longrightarrow \int_{Z}^{\oplus} L_{2}(D, H(z)) \mathrm{d} z \cong \int_{Z}^{\oplus} H(z) \mathrm{d} z \otimes L_{2}(D)
$$


the so-called Floquet or partial Fourier transformation. Moreover, operators commuting with the translation $T$ on $L_{2}(X)$ are decomposable, in particular, we can decompose $\Delta_{X}$ since its resolvent commutes with all translations (2.2). One can show in this context (cf. [Sun88, LP04]):

Theorem 4.5. The operator $U$ maps $C_{\mathrm{c}}^{\infty}(X)$ into $\int_{Z}^{\oplus} C_{\mathrm{eq}}^{\infty}(D, H(z)) \mathrm{d} z$. Moreover, $\Delta_{X}$ is unitary equivalent to $\int_{Z}^{\oplus} \Delta_{D}^{\mathrm{eq}}(z) \mathrm{d} z$ and

$$
\operatorname{spec} \Delta_{X} \subseteq \overline{\bigcup_{z \in Z} \operatorname{spec} \Delta_{D}^{\mathrm{eq}}(z)}
$$

We denote by $C_{\mathrm{eq}}^{\infty}(D, H(z))$ the set of smooth $R(z)$-equivariant functions defined in (4.1) and $\Delta_{D}^{\mathrm{eq}}(z)$ is the $R(z)$-equivariant Laplacian in $L_{2}(D, H(z))$.

If $\Gamma$ is amenable (cf. Remark 5.3), then we have equality in (4.10).

Example 4.6. Let us illustrate the above direct integral decomposition in the case of the free group $\Gamma=\mathbb{Z} * \mathbb{Z}$ generated by $\alpha$ and $\beta$. Let $A \cong \mathbb{Z}$ be the cyclic subgroup generated by $\alpha$. We can decompose the algebra $\mathcal{R}$ given in (4.8) w.r.t. the abelian algebra $\mathcal{A}:=\left\{L_{a} \in \mathcal{L}\left(\ell_{2}(\Gamma)\right) \mid a \in A\right\} \subset \mathcal{R}^{\prime}$, and, in this case, we have $Z=\mathbb{S}^{1}$. Since the set $\left\{a \gamma a^{-1} \mid a \in A\right\}$ is infinite provided $\gamma \notin A$, the algebra is maximal abelian in $\mathcal{R}^{\prime}$ (i.e., $\mathcal{A}=\mathcal{A}^{\prime} \cap \mathcal{R}^{\prime}$ ), and therefore, each fibre representation $R(z)$ is irreducible in $\mathcal{H}(z)$. Moreover, since $L_{a} \in \mathcal{A}^{\prime}(a \in A)$ we can also decompose these operators w.r.t the previous direct integral.

We can give a more concrete realisation of the abstract Fourier transformation $F=F_{\Gamma}$ (see e.g. [Rob83, Section 19]): We interprete $\Gamma \rightarrow A \backslash \Gamma$ as covering space with abelian covering group $A$ acting on $\Gamma$ from the left; the corresponding translation action $T_{a}$ on $\ell_{2}(\Gamma)$ coincides with the left regular representation $L_{a}$ $(a \in A)$. The (abelian) Floquet transformation $U=U_{A}$ gives a direct integral decomposition

$$
F_{\Gamma}=U_{A}: \ell_{2}(\Gamma) \longrightarrow \int_{\widehat{A}}^{\oplus} H(\chi) \mathrm{d} \chi,
$$

where $H(\chi) \cong \ell_{2}(A \backslash \Gamma)$ is the space of $\chi$-equivariant sequences in $\ell_{2}(\Gamma)$. Note that $H(\chi)$ is infinite dimensional. A straightforward calculation shows that

$$
R_{\gamma} \cong \int_{\widehat{A}}^{\oplus} R_{\gamma}(\chi) \mathrm{d} \chi \quad \text { and } \quad L_{a} \cong \int_{\widehat{A}}^{\oplus} L_{a}(\chi) \mathrm{d} \chi,
$$

where $R_{\gamma}(\chi) u(\widetilde{\gamma})=u(\widetilde{\gamma} \gamma)$ and $L_{a}(\chi) u(\widetilde{\gamma})=\bar{\chi}(a) u(\widetilde{\gamma})$ for $u \in H(\chi)$. Furthermore, one sees that $v=\left(U \delta_{e}\right)(\chi)$ is the unique normalised eigenvector of $R_{a}(\chi)$ with eigenvalue $\chi(a)$. This follows from the fact that the set of cosets $\{A \gamma a \mid a \in$ $A\} \subset A \backslash \Gamma$ is infinite provided $\gamma \notin A$. From the previous facts one can directly check that each $R(\chi)$ is an irreducible representation of $\Gamma$ in $H(\chi)$ and that these representations are mutually inequivalent. Finally, $R(\chi)$ is also inequivalent to any irreducible component of the direct integral decomposition obtained from a different maximal abelian subgroup $B \neq A$. 


\section{Spectral Gaps FOR TyPe I GROUPS}

We will present in this section the first method to show that the Laplacian of the manifolds constructed in Section 3 with (in general non-abelian) type I covering groups have an arbitrary finite number of spectral gaps. We begin recalling the definition of type I groups in the context of discrete groups.

Definition 5.1. A discrete group $\Gamma$ is of type $I$ if $\Gamma$ is a finite extension of an abelian group, i.e., if there is an exact sequence

$$
0 \longrightarrow A \longrightarrow \Gamma \longrightarrow \Gamma_{0} \longrightarrow 0
$$

where $A \triangleleft \Gamma$ is abelian and $\Gamma_{0} \cong \Gamma / A$ is a finite group.

Remark 5.2. (1) In the previous definition we have used a simple characterisation of countable, discrete groups of type I due to Thoma, cf. [Tho64] (for uncountable groups see [Tho68]). Moreover, all irreducible representations of a type I group $\Gamma$ are finite-dimensional and have a uniform bound on the dimension (see [Tho64, Moo72]). Therefore, the following properties are all equivalent: (a) there is a uniform bound on the dimensions of irreducible representations of $\Gamma$, (b) all irreducible representations of $\Gamma$ are finite-dimensional, (c) $\Gamma$ is a finite extension of an abelian group, (d) $\Gamma$ is CCR (completely continuous representation, cf. [Wal92, Ch. 14]), (e) $\Gamma$ is of type I. Recall also that $\Gamma$ is of type I iff the von Neumann algebra $\mathcal{R}$ generated by $\Gamma$ (cf. Eq. (4.8)) is of type $I$ (cf. [Kan69]).

Note that for our application it would be enough if $\Gamma$ has a decomposition over a measure space $(Z, \mathrm{~d} z)$ as in Remark 4.4 such that almost every representation $\rho(z)$ is finite-dimensional. But such a group is already of type I: indeed, if the set $\{z \in Z \mid \operatorname{dim} H(z)=\infty\}$ has measure 0 , then it follows from [Dix81, Section II.3.5] that the von Neumann Algebra $\mathcal{R}$ (cf. Eq. (4.8)) is of type I. By the above equivalent characterisation this implies that $\Gamma$ is of type $\mathrm{I}$.

(2) The following criterion (cf. [Kan69, Kal70]) will be used in Examples 8.4 and 8.5 to decide that a group is not of type $\mathrm{I}$ : The von Neumann algebra $\mathcal{R}$ is of type $\mathrm{II}_{1}$ iff $\Gamma_{\text {fcc }}$ has infinite index in $\Gamma$. Here,

$$
\Gamma_{\mathrm{fcc}}:=\left\{\gamma \in \Gamma \mid C_{\gamma} \text { is finite }\right\}
$$

is the set of elements $\gamma \in \Gamma$ having finite conjugacy class $C_{\gamma}$. In particular such a group is not of type $\mathrm{I}$. Even worse: Almost all representations in the direct integral decomposition (4.7) are of type $\mathrm{II}_{1}$ ([Dix81, Section II.3.5]) and therefore infinite-dimensional (see e.g. Example 4.6).

Remark 5.3. The notion of amenable discrete group will be useful at different stages of our approach. For a definition of amenability of a discrete group $\Gamma$ see e.g. [Day57] or [Bro81]. We will only need the following equivalent characterisations: (a) $\Gamma$ is amenable. (b) $0 \in \operatorname{spec} \Delta_{X}$ [Bro81]. (c) $\operatorname{spec} \Delta_{M} \subset$ 
spec $\Delta_{X}$ [Sun88, Propositions 7-8]. Here, $X \rightarrow M$ is a covering with covering group $\Gamma$. Note that discrete type I groups are amenable since they are finite extensions of abelian groups (extensions of amenable groups are again amenable, cf. [Day57, Section 4]).

We want to stress that Theorem 3.3 is no contradiction to the fact that $\Gamma$ is amenable iff $0 \in \operatorname{spec} \Delta_{\left(X, g_{\varepsilon}\right)}$ although the first interval $I_{1}\left(g_{\varepsilon}\right)=\left[0, \lambda_{k}^{+}\left(g_{\varepsilon}\right)\right]$ tends to 0 as $\varepsilon \rightarrow 0$. Note that we have only shown there that $I_{1}\left(g_{\varepsilon}\right) \cap \operatorname{spec} \Delta_{\left(X, g_{\varepsilon}\right)} \neq \emptyset$ and not $0=\lambda_{1}\left(M, g_{\varepsilon}\right) \in \operatorname{spec} \Delta_{\left(X, g_{\varepsilon}\right)}$ which is only true in the amenable case.

The dual of $\Gamma$, which we denote by $\widehat{\Gamma}$, is the set of equivalence classes of unitary irreducible representations of $\Gamma$. We denote by $[\rho]$ the (unitary) equivalence class of a unitary representation $\rho$ on $H$. Note that the spectrum of a $\rho$-equivariant Laplacian and $\operatorname{dim} H$ only depend on the equivalence class of $\rho$.

If $\Gamma$ is of type I, then the dual $\widehat{\Gamma}$ becomes a nice measure space ("smooth" in the terminology of [Mac76, Chapter 2]). Furthermore, we can use $\widehat{\Gamma}$ as measure space in the direct integral decomposition defined in Subsection 4.3. In particular, combining the results of Section 2 and 4 we obtain the main result for type I groups:

Theorem 5.4. Suppose $X \rightarrow M$ is a Riemannian $\Gamma$-covering with fundamental domain $D$, where $\Gamma$ is a type I group and denote by $g$ the Riemannian metric on $X$. Then

$$
\operatorname{spec} \Delta_{(X, g)} \subset \bigcup_{k \in \mathbb{N}} I_{k}(g), \quad \text { and } \quad I_{k}(g) \cap \operatorname{spec} \Delta_{(X, g)} \neq \emptyset, \quad k \in \mathbb{N},
$$

where $I_{k}(g):=\left[\lambda_{k}^{-}(D, g), \lambda_{k}^{+}(D, g)\right]$ is the Neumann-Dirichlet interval defined as in (3.2). In particular, for each $n \in \mathbb{N}$ there exists a metric $g=g_{\varepsilon_{n}}$ constructed as in Subsection 3.2 such that $\operatorname{spec} \Delta_{(X, g)}$ has at least $n$ gaps, i.e., $n+1$ components as subset of $[0, \infty)$.

Proof. We have

$$
\operatorname{spec} \Delta_{X}=\overline{\bigcup_{[\rho] \in \widehat{\Gamma}} \operatorname{spec} \Delta_{D, H}^{\rho}} \subseteq \overline{\bigcup_{k \in \mathbb{N}} I_{k}(g)}=\bigcup_{k \in \mathbb{N}} I_{k}(g),
$$

where we used the Theorem 4.5 with $Z=\widehat{\Gamma}$ for the first equality and Theorem 4.3 for the inclusion. Note that $\Gamma$ is amenable and that the latter theorem applies since all (equivalence classes of) irreducible representations of a type I group are finite-dimensional (cf. Remark $5.2(1)$ ). The existence of gaps in $\bigcup_{k} I_{k}(g)$ follows from Theorem 3.2.

Since $\Gamma$ is amenable, spec $\Delta_{M} \subset$ spec $\Delta_{X}$ (cf. (c) in Remark 5.3). Moreover, from Eq. (4.4) with $\rho$ the trivial representation on $\mathcal{H}=\mathbb{C}$, we have that $\lambda_{k}(M) \in I_{k}$. Note that functions on $M$ correspond to functions on $D$ with periodic boundary conditions. Therefore, we have shown that every gap of the union $\bigcup_{k} I_{k}(g)$ is also a gap of $\operatorname{spec} \Delta_{X}$. 


\section{Spectral Gaps FOR Residually Finite Groups}

In this section, we present a new method to prove the existence of a finite number of spectral gaps of $\Delta_{X}$. The present approach is applicable to so-called residually finite groups $\Gamma$, which is a much larger class of groups containing type I groups (cf. Section 8). Roughly speaking, residually finite means that $\Gamma$ has a lot of normal subgroups with finite index. Geometrically, this implies that one can approximate the covering $\pi: X \longrightarrow M$ with covering transformation group $\Gamma$ by finite coverings $p_{i}: M_{i} \longrightarrow M$, where the $M_{i}$ 's are compact.

Since the present section is central to the paper we will also give for completeness proofs of known results (for Theorem 6.6 see also [ASS94, Ada95])

6.1. Subcoverings and residually finite groups. Suppose that $\pi: X \longrightarrow$ $M$ is a covering with covering transformation group $\Gamma$ (as in Subsection 2.1). Corresponding to a normal subgroup $\Gamma_{i} \triangleleft \Gamma$ we associate a covering $\pi_{i}: X \longrightarrow M_{i}$ such that

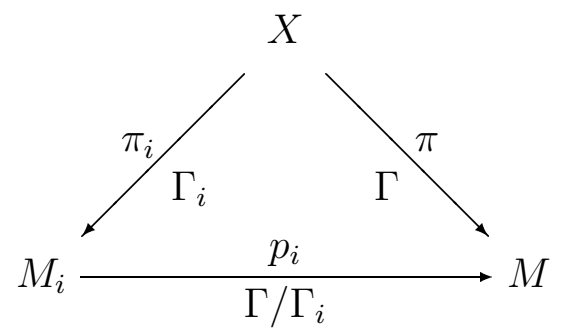

is a commutative diagram. The groups under the arrows denote the corresponding covering transformation groups.

Definition 6.1. A (countable, infinite) discrete group $\Gamma$ is residually finite if there exists a monotonous decreasing sequence of normal subgroups $\Gamma_{i} \triangleleft \Gamma$ such that

$$
\Gamma=\Gamma_{0} \triangleright \Gamma_{1} \triangleright \cdots \triangleright \Gamma_{i} \triangleright \cdots, \bigcap_{i \in \mathbb{N}} \Gamma_{i}=\{e\} \quad \text { and } \quad \Gamma / \Gamma_{i} \text { is finite. }
$$

Denote by $\mathfrak{R} \mathcal{F}$ the class of residually finite groups.

Suppose now that $\Gamma$ is residually finite. Then there exists a corresponding sequence of coverings $\pi_{i}: X \longrightarrow M_{i}$ such that $p_{i}: M_{i} \longrightarrow M$ is a finite covering (cf. Diagram (6.1)). Such a sequence of covering maps is also called tower of coverings.

Remark 6.2. We recall also the following equivalent definitions of residually finite groups (see e.g. [Mag69] or [Rob82, Section 2.3]).

(i) A group $\Gamma$ is called residually finite if for all $\gamma \in \Gamma \backslash\{e\}$ there is a group homomorphism $\Psi: \Gamma \longrightarrow G$ such that $\Psi(\gamma) \neq e$ and $\Psi(\Gamma)$ is a finite group. 
(ii) Let $\mathcal{F}$ denote the class of finite groups. $\Gamma$ is residually finite, if the socalled $\mathcal{F}$-residual

$$
\mathfrak{R}_{\mathcal{F}}(\Gamma):=\bigcap_{\substack{N \triangleleft \Gamma \\ \Gamma / N \in \mathcal{F}}} N
$$

is trivial, i.e., $\mathfrak{R}_{\mathcal{F}}(\Gamma)=\{e\}$.

Next we give some examples for residually finite groups (cf. the survey article [Mag69]):

Example 6.3. (i) Abelian and finite groups are residually finite. (ii) Free products of residually finite groups are residually finite, in particular, the free group in $r$ generators $\mathbb{Z}^{* r}$ is residually finite. (iii) Finitely generated linear groups are residually finite (for a simple proof of this fact cf. [Alp87]; a group is called linear iff it is isomorphic to a subgroup of $\mathrm{GL}_{n}(\mathbb{C})$ for some $n \in \mathbb{N}$.) In particular, $\mathrm{SL}_{n}(\mathbb{Z})$ and fundamental groups of closed, orientable surfaces of genus $g$ are residually finite.

Next we need to introduce a metric on the discrete space $\Gamma$ :

Definition 6.4. Let $E$ be a set which generates $\Gamma$. The word metric $d=d_{E}$ on $\Gamma$ is defined as follows: $d(\gamma, e)$ is the minimal number of elements in $E$ needed to express $\gamma$ as a word in the alphabet $E ; d(e, e):=0$ and $d(\gamma, \widetilde{\gamma}):=d\left(\gamma \widetilde{\gamma}^{-1}, e\right)$.

Geometrically, residually finiteness means that, given any compact set $K \subset X$, there exists a finite covering $p_{i}: M_{i} \longrightarrow M$ and a covering $\pi_{i}: X \longrightarrow M_{i}$ which is injective on $K$ (cf. [Bro86]). This idea is used in the following lemma:

Lemma 6.5. Fix a fundamental domain $D$ for the covering $\pi: X \longrightarrow M$. Then for each covering $\pi_{i}: X \longrightarrow M_{i}$ there is a fundamental domain $D_{i}$ (not necessarily connected) such that

$$
D_{0}:=D \subset D_{1} \subset \cdots \subset D_{i} \subset \cdots \quad \text { and } \quad \bigcup_{i \in \mathbb{N}} D_{i}=X .
$$

Proof. It is enough to show the existence of a family of representants $R_{i} \subset \Gamma$ of $\Gamma / \Gamma_{i}, i \in \mathbb{N}$, satisfying

$$
R_{0}:=\{e\} \subset R_{1} \subset \cdots \subset R_{i} \subset \cdots \quad \text { and } \bigcup_{i \in \mathbb{N}} R_{i}=\Gamma .
$$

In this case the fundamental domains are given explicitly by

$$
D_{i}:=\operatorname{int} \bigcup_{r \in R_{i}} r^{-1} \bar{D}
$$

where int denotes the topological interior.

Let $d$ be the word metric on $\Gamma$ with respect to the set of generators $E:=\{\gamma \in$ $\Gamma \mid \gamma \bar{D} \cap \bar{D} \neq \emptyset\}$, which is naturally adapted to the fundamental domain $D$. Note 
that $E$ is finite and generates $\Gamma$ since $\bar{D}$ is compact (cf. [Rat94, Thms. 6.5.10 and 6.5.11]).

We choose a set of representants $R_{i}$ of $\Gamma / \Gamma_{i}$ that have minimal distance in the word metric to the neutral element, i.e. if $r \in R_{i}$, then $d(r, e) \leq d\left(r \Gamma_{i}, e\right)$. Note that since $\Gamma_{i+1} \subset \Gamma_{i}$ we have $R_{i+1} \supset R_{i}$. To conclude the proof we have to show that every $\gamma \in \Gamma$ is contained in some $R_{i}, i \in \mathbb{N}$. Since $\Gamma$ is finitely generated, there exists $n \in \mathbb{N}$ such that $\gamma \in B_{n}:=\{\gamma \in \Gamma \mid d(\gamma, e) \leq n\}$. Moreover, since $B_{2 n}$ is finite and $\Gamma$ residually finite we also have $B_{2 n} \cap \Gamma_{i}=\{e\}$ for $i$ large enough. Therefore, any other element $\widetilde{\gamma}=\gamma \gamma_{i}^{-1}$ in the class $\gamma \Gamma_{i}$ with $\gamma_{i} \in \Gamma_{i} \backslash\{e\}$ has a distance greater than $n$, since

$$
d(\widetilde{\gamma}, e)=d\left(\gamma \gamma_{i}^{-1}, e\right)=d\left(\gamma, \gamma_{i}\right) \geq d\left(e, \gamma_{i}\right)-d(\gamma, e)>2 n-n=n .
$$

This implies that $\gamma \in R_{i}$ by the minimality condition in the choice of the representants.

Theorem 6.6. Suppose $\Gamma$ is residually finite with the associated sequence of coverings $\pi_{i}: X \longrightarrow M_{i}$ and $p_{i}: M_{i} \longrightarrow M$ as in (6.1). Then

$$
\operatorname{spec} \Delta_{X} \subseteq \overline{\bigcup_{i \in \mathbb{N}} \operatorname{spec} \Delta_{M_{i}}},
$$

and the Laplacian $\Delta_{M_{i}}$ w.r.t. the finite covering $p_{i}: M_{i} \longrightarrow M$ has discrete spectrum. Equality holds iff $\Gamma$ is amenable.

Proof. (Cf. [Ada95]) If $\lambda \in \operatorname{spec} \Delta_{X}$, then for each $\varepsilon>0$ there exists $u \in C_{\mathrm{c}}^{\infty}(X)$ such that

$$
\frac{\left\|\left(\Delta_{X}-\lambda\right) u\right\|_{X}^{2}}{\|u\|_{X}^{2}}<\varepsilon .
$$

Applying Lemma 6.5 there is an $i=i(\varepsilon)$ such that supp $u \subset D_{i}$. Furthermore, since $D_{i} \hookrightarrow M_{i}=X / \Gamma_{i}$ is an isometry, $u$ can be written as the lift of a smooth $f$ on $M_{i}$, i.e., $f \circ \pi_{i}=u$. Therefore,

$$
\frac{\left\|\left(\Delta_{M_{i}}-\lambda\right) f\right\|_{M_{i}}^{2}}{\|f\|_{M_{i}}^{2}}=\frac{\left\|\left(\Delta_{X}-\lambda\right) u\right\|_{X}^{2}}{\|u\|_{X}^{2}}<\varepsilon,
$$

which implies $\lambda \in \overline{\bigcup_{i \in \mathbb{N}} \operatorname{spec} \Delta_{M_{i}}}$. Finally, since $M_{i} \rightarrow M$ is a finite covering and $M$ is compact, spec $\Delta_{M_{i}}$ is discrete. For the second assertion cf. [Ada95] or [ASS94]. One basically uses the characterisation due to [Bro81] that $\Gamma$ is amenable iff $0 \in \operatorname{spec} \Delta_{X}$ (cf. Remark 5.3).

Next we analyse the spectrum of the finite covering $M_{i} \rightarrow M$. Note that $D$ is also isometric to a fundamental domain for each finite covering $M_{i} \rightarrow M, i \in \mathbb{N}$.

Lemma 6.7. We have

$$
\operatorname{spec} \Delta_{M_{i}}=\bigcup_{[\rho] \in \widehat{G_{i}}} \operatorname{spec} \Delta_{D, H(\rho)}^{\rho},
$$


where $\Delta^{\rho}$ is the equivariant Laplacian introduced in Subsection 4.1 and $G_{i}:=\Gamma / \Gamma_{i}$ is a finite group and $\widehat{G_{i}}$ its dual.

Proof. Applying the results of Subsection 4.3 to the finite group $G_{i}$ and the finite measure space $Z:=\widehat{G_{i}}$ with the counting measure all direct integrals become direct sums. By Peter-Weyl's theorem (see e.g. [HR70, §27.49]) we also have

$$
F: \ell_{2}\left(G_{i}\right) \longrightarrow \bigoplus_{[\rho] \in \widehat{G_{i}}} n(\rho) H(\rho),
$$

where each multiplicity satisfies $n(\rho)=\operatorname{dim} H(\rho)<\infty$. Finally,

$$
\Delta_{M_{i}} \cong \bigoplus_{[\rho] \in \widehat{G_{i}}} \Delta_{D, H(\rho)}^{\rho}
$$

and the result follows.

We now can formulate the main result of this section:

Theorem 6.8. Suppose $X \rightarrow M$ is a Riemannian $\Gamma$-covering with fundamental domain $D$, where $\Gamma$ is a residually finite group and denote by $g$ the Riemannian metric on $X$. Then

$$
\operatorname{spec} \Delta_{(X, g)} \subset \bigcup_{k \in \mathbb{N}} I_{k}(g), \quad I_{k}(g) \cap \operatorname{spec} \Delta_{(X, g)} \neq \emptyset, \quad k \in \mathbb{N},
$$

where $I_{k}(g):=\left[\lambda_{k}^{-}(D, g), \lambda_{k}^{+}(D, g)\right]$ is defined as in (3.2). In particular, for each $n \in \mathbb{N}$ there exists a metric $g=g_{\varepsilon_{n}}$, constructed as in Subsection 3.2, such that $\operatorname{spec} \Delta_{(X, g)}$ has at least $n$ gaps, i.e., $n+1$ components as subset of $[0, \infty)$.

Proof. We have

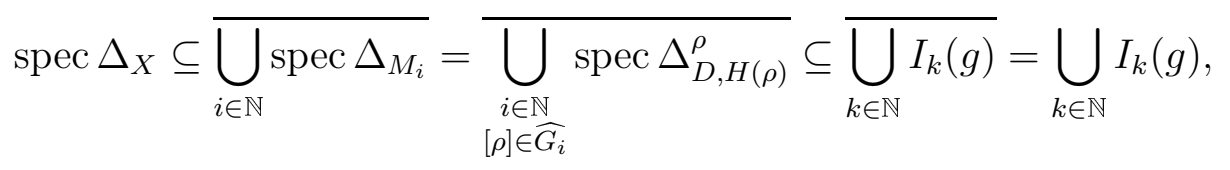

where we used Theorem 6.6, Lemma 6.7 and Theorem 4.3. Note that the latter theorem applies since all (equivalence classes of) irreducible representations of the finite groups $G_{i}, i \in \mathbb{N}$, are finite-dimensional. The existence of gaps in $\bigcup_{k} I_{k}(g)$ follows from Theorem 3.2. Finally, by Theorem 3.3, a gap of $\bigcup_{k} I_{k}(g)$ is in fact a gap of $\operatorname{spec} \Delta_{X}$.

\section{Kadison CONSTANT AND ASYMPtotic BEHAVIOUR}

In the present section we will combine our main result stated in Theorem 6.8 with some results by Sunada and Brüning (cf. [Sun92, Theorem 1] or [BS92]), to give a more complete description of the spectrum of the Laplacian $\Delta_{X}$, where $X \rightarrow M$ is the covering manifold constructed in Section 3. For this, we need a further definition: 
Definition 7.1. The Kadison constant of $\Gamma$ is defined as

$$
C(\Gamma):=\inf \left\{\operatorname{tr}_{\Gamma}(P) \mid P \text { non-trivial projection in } C_{\text {red }}^{*}(\Gamma, \mathcal{K})\right\},
$$

where $\operatorname{tr} \Gamma(\cdot)$ is the canonical trace on $C_{\text {red }}^{*}(\Gamma, \mathcal{K})$, the tensor product of the reduced group $C^{*}$-algebra of $\Gamma$ and the algebra $\mathcal{K}$ of compact operators on a separable Hilbert space of infinite dimension (see [Sun92, Section 1] for more details.)

In this section, we assume that $\Gamma$ is is residually finite and has a strictly positive Kadison constant, i.e. $C(\Gamma)>0$. For example, the free product $\mathbb{Z}^{* r} * \Gamma_{1} * \cdots * \Gamma_{a}$ with finite groups $\Gamma_{i}$ satisfies both properties (cf. e.g. [Mag69], [Sun92, Appendix]). Another such group is the fundamental group (cf. Eq. (3.1)) of a (compact, orientable) surface of genus $g$ (see [MM99]).

Remark 7.2. Suppose that $K$ is an integral operator on $L_{2}(X)$ commuting with the group action, having smooth kernel $k(x, y)$ and satisfying

$$
k(x, y)=0 \quad \text { for all } x, y \in X \text { with } d(x, y) \geq c
$$

for some constant $c>0$. Then $K$ can be interpreted as an element of $C_{\text {red }}^{*}(\Gamma, \mathcal{K})$ and one can write the $\Gamma$-trace as

$$
\operatorname{tr}_{\Gamma} K=\int_{D} k(x, x) d x
$$

(see [Sun92, Section 1] as well as [Ati76] for further details), where $D$ is a fundamental domain of $X \rightarrow M$.

If we consider the spectral resolution of the Laplacian $\Delta_{X} \cong \int^{\oplus} \lambda \mathrm{d} E(\lambda)$, then it follows that

$$
E\left(\lambda_{2}\right)-E\left(\lambda_{1}\right) \in C_{\text {red }}^{*}(\Gamma, \mathcal{K})
$$

if $\lambda_{1}<\lambda_{2}$ and $\lambda_{1}, \lambda_{2} \notin \operatorname{spec} \Delta_{X}$ (cf. [Sun92, Section 2]).

Theorem 7.3. Suppose $(X, g) \rightarrow(M, g)$ is a Riemannian $\Gamma$-covering where $\Gamma$ is a residually finite group having positive Kadison constant, i.e., $C(\Gamma)>0$. Denote by $\mathcal{N}(g, \lambda)$ the number of components of spec $\Delta_{(X, g)}$ which intersect the interval $[0, \lambda]$. Then we have

(i) For each $n \in \mathbb{N}$ there exists a metric $g=g_{\varepsilon_{n}}$ constructed as in Subsection 3.2 such that spec $\Delta_{(X, g)}$ has at least $n$ gaps, i.e., $\mathcal{N}\left(g_{\varepsilon_{n}}, \infty\right) \geq n+1$.

(ii) $\operatorname{spec} \Delta_{(X, g)}$ has band structure, i.e. it is a locally finite union of closed intervals. In other words, $\mathcal{N}(g, \lambda)<\infty$ for all $\lambda \geq 0$.

(iii) The following asymptotic upper bound holds:

$$
\limsup _{\lambda \rightarrow \infty} \frac{\mathcal{N}(g, \lambda)}{(2 \pi)^{-d} \omega_{d} \operatorname{vol}(M, g) \lambda^{d / 2}} \leq \frac{1}{C(\Gamma)} .
$$


(iv) Suppose in addition that the limit manifold $(N, g)$ has simple spectrum, i.e., all eigenvalues $\lambda_{k}(0)$ have multiplicity 1 (cf. Theorem 3.1). Then for each $\lambda \geq 0$ there exists $\varepsilon(\lambda)>0$ such that

$$
\liminf _{\lambda \rightarrow \infty} \frac{\mathcal{N}\left(g_{\varepsilon(\lambda)}, \lambda\right)}{(2 \pi)^{-d} \omega_{d} \operatorname{vol}(N, g) \lambda^{d / 2}} \geq 1
$$

Here, $g_{\varepsilon}$ denote the metric constructed in Section 3.

Proof. (i) follows immediately from Theorem 6.8. (ii) and (iii) have been shown in [Sun92] or [BS92]. (iv) Suppose $\lambda \notin \operatorname{spec} \Delta_{N}$, then $\lambda_{k}(0)<\lambda<\lambda_{k+1}(0)$ for some $k \in \mathbb{N}$. Let $\varepsilon=\varepsilon(\lambda) \in(0,1]$ be the largest number such that $\mathcal{N}\left(\lambda, g_{\varepsilon}\right)$ is (at least) $k$, in other words, $\mathcal{N}\left(\lambda, g_{\varepsilon}\right) \geq k=\mathcal{N}\left(\lambda, \Delta_{N}\right)$ where the latter number denotes the number of eigenvalues of $\Delta_{N}$ below $\lambda$. By the Weyl theorem,

$$
\lim _{\lambda \rightarrow \infty} \frac{\mathcal{N}\left(\lambda, \Delta_{N}\right)}{(2 \pi)^{-d} \omega_{d} \operatorname{vol}(N, g) \lambda^{d / 2}}=1
$$

and the result follows.

Remark 7.4. Note that (iii) only gives an asymptotic upper bound on the number of components of spec $\Delta_{X} \cap[0, \lambda]$, not on the whole spectrum itself. Therefore, we have no assertion about the so-called Bethe-Sommerfeld conjecture stating that the number of spectral gaps for a periodic operator in dimensions $d \geq 2$ remains finite.

To conclude the section we remark that that generically, $\Delta_{(N, g)}$ has simple spectrum (cf. [Uhl76]). The assumption in (iv) is natural since $\mathcal{N}(g, \lambda)$ counts the components without multiplicity.

\section{EXAMPLES}

8.1. Relation between the approaches presented in Sections 5 and 6. We begin comparing the two main approaches presented in this paper which assure the existence of spectral gaps (cf. Sections 5 and 6).

One easily sees from Definition 6.1 that a finite extension of a residually finite group is again residually finite. In particular, type I groups are residually finite as finite extensions of abelian groups (cf. Definition 5.1). Therefore, for type I groups one can also produce spectral gaps by the approximation method with finite coverings introduced in Section 6. Nevertheless we believe that the direct integral method will be useful when analysing further spectral properties:

Example 8.1. One of the advantages of the method described in Section 5 is that one has more information about the bands. Suppose $\Gamma$ is finitely generated and abelian, i.e. $\Gamma \cong \mathbb{Z}^{r} \oplus \Gamma_{0}$, where $\Gamma_{0}$ is the torsion subgroup of $\Gamma$. Then $\widehat{\Gamma}$ is the disjoint union of finitely many copies of $\mathbb{T}^{r}$. From the continuity of the 
map $\rho \rightarrow \lambda_{k}^{\rho}$ (cf. [BJR99] or [Sun90]), we can simplify the characterisation of the spectrum in Theorem 4.5 and obtain

$$
\operatorname{spec} \Delta_{X}=\bigcup_{k \in \mathbb{N}} B_{k}, \quad \text { where } \quad B_{k}:=\left\{\lambda_{k}^{\rho} \mid \rho \in \widehat{\Gamma}\right\} \subseteq I_{k},
$$

the $k$-th band. Since $\widehat{\Gamma}$ is compact, $B_{k}$ is also compact, but in general, $B_{k}$ need not to be connected (recall that $\widehat{\Gamma}$ is connected iff $\Gamma$ is torsion free, i.e., $\Gamma=\mathbb{Z}^{r}$ ). Note also that $B_{k}$ has only finitely many components. For non-abelian groups this approach may be generalised in the direction of Hilbert $\mathrm{C}^{*}$-modules (cf. [Gru01]).

In principle one could also consider a combination of the methods of Section 5 and 6: denote by $\mathcal{T}_{1}$ the class of type I groups and by $\mathfrak{R} \mathcal{T}_{1}$ the class of residually type $I$ groups, i.e., $\Gamma \in \mathfrak{R} \mathcal{T}_{1}$ iff the $\mathcal{T}_{1}$-residual $\mathfrak{R}_{\mathcal{T}_{1}}(\Gamma)$ is trivial (cf. Eq. (6.3)). Similarly we denote by $\mathfrak{R} \mathcal{F}$ the class of residually finite groups (cf. Definition 6.1). If we consider a covering with a group $\Gamma \in \mathfrak{R} \mathcal{T}_{1}$, then instead of the finite covering $p_{i}: M_{i} \longrightarrow M$ considered in Eq. (6.1) we would have a covering with a type I group. For these groups, we can replace Lemma 6.7 by the direct integral decomposition of Theorem 4.5. Nevertheless the following lemma shows that the class of residually finite and residually type I groups coincide.

Lemma 8.2. From the inclusion $\mathcal{F} \subset \mathcal{T}_{1} \subset \mathfrak{R} \mathcal{F}$ it follows that the corresponding residuals for the group $\Gamma$ coincide, i.e., $\mathfrak{R}_{\mathcal{F}}(\Gamma)=\mathfrak{R}_{\mathcal{T}_{1}}(\Gamma)$. Moreover, $\mathfrak{R} \mathcal{F}=\mathfrak{R} \mathcal{T}_{1}$.

Proof. From the inclusion $\mathcal{F} \subset \mathcal{T}_{1}$ it follows immediately that $\mathfrak{R}_{\mathcal{F}}(\Gamma) \supset \mathfrak{R}_{\mathcal{T}_{1}}(\Gamma)$. To show the reverse inclusion one uses the following characterisation: a group is residually $\mathcal{F}$ iff it is a subcartesian product of finite groups (cf. [Rob82, § 2.3.3]). Finally, from the equality of the residuals it follows that $\mathfrak{R F}=\mathfrak{R} \mathcal{T}_{1}$.

8.2. Examples with residually finite groups. In the rest of this subsection we present several examples of residually finite groups which are not type I. They show different aspects of our analysis.

For the next example recall the construction procedure (A) described in Section 3.

Example 8.3 (Fundamental groups of closed surfaces). Suppose that $N:=\mathbb{S}^{2}$ is the two-dimensional sphere with a metric such that $\Delta_{N}$ has simple spectrum (cf. [Uhl76] for the existence of such metrics). Suppose, in addition, that $M$ is obtained by adding $r$ handles to $N$ as described in Section 3, Case A. The fundamental group $\Gamma$ of $M$ (cf. Eq. (3.1) with $s=0$ ) is residually finite (recall Example 6.3 (iii)). Moreover, from the proof of Proposition 2.16 in [MM99], $\Gamma$ has a positive Kadison constant. Therefore, Theorem 7.3 applies to the the universal cover $X:=\widetilde{M} \rightarrow M$ with the metric $g_{\varepsilon}$ specified in Section 3 .

The following example uses the construction procedure (B) in Section 3. 
Example 8.4 (Heisenberg group). Let $\Gamma:=H_{3}(\mathbb{Z})$ be the discrete Heisenberg group, where $H_{3}(R)$ denotes the set of matrices

$$
A_{x, y, z}:=\left(\begin{array}{ccc}
1 & x & y \\
0 & 1 & z \\
0 & 0 & 1
\end{array}\right)
$$

with coefficients $x, y, z$ in the ring $R$. A covering with group $\Gamma$ is given e.g. by $X:=H_{3}(\mathbb{R})$ with compact quotient $M:=H_{3}(\mathbb{R}) / H_{3}(\mathbb{Z})$. Note that $X$ is diffeomorphic to $\mathbb{R}^{3}$. Clearly, $\Gamma$ is a finitely generated linear group and therefore residually finite (cf. Example 6.3 (iii)). Now, by Theorem 6.8 one can deform conformally a $\Gamma$-invariant metric $g$ as in Case (B) of Section 3, such that spec $\Delta_{X}$ has at least $n$ spectral gaps, $n \in \mathbb{N}$.

In this case, $\Gamma$ is also amenable as an extension of amenable groups (cf. Remark 5.3). In fact, $\Gamma$ is isomorphic to the semi-direct product $\mathbb{Z} \ltimes \mathbb{Z}^{2}$, where $1 \in \mathbb{Z}$ acts on $\mathbb{Z}^{2}$ by the matrix

$$
\left(\begin{array}{ll}
1 & 1 \\
0 & 1
\end{array}\right)
$$

Therefore, we have equality in the characterisation of $\operatorname{spec} \Delta_{X}$ in Theorems 4.5 and 6.6 .

Note finally that the group $\Gamma$ is not of type I since $\Gamma_{\text {fcc }}=\left\{A_{0, y, 0} \mid y \in \mathbb{Z}\right\}$ has infinite index in $\Gamma$ (cf. Remark $5.2(2)$ ). Thus, our method in Section 5 does not apply since the measure $\mathrm{d} z$ in (4.6) is supported only on infinite-dimensional Hilbert spaces. Curiously, one can construct a finitely additive measure on the group dual $\widehat{\Gamma}$ supported by the set of finite-dimensional representations of $\widehat{\Gamma}$ (cf. [Pyt79]). The group dual $\widehat{\Gamma}$ is calculated e.g. in [Kan68, Beispiel 1].

Example 8.5 (Free groups). Let $\Gamma=\mathbb{Z}^{* r}$ be the free group with $r>1$ generators. Then $\Gamma$ is residually finite (recall Example 6.3 (ii)) and has positive Kadison constant (cf. [Sun92, Appendix]). Therefore, Theorem 7.3 applies to the $\Gamma$-coverings $X \rightarrow M$ specified in Section 3 .

Note that $\Gamma$ is not of type I since $\Gamma_{\text {fcc }}=\{e\}$ (cf. Remark $5.2(2)$ ). Such groups are called ICC (infinite conjugacy class) groups. Again, for any direct integral decomposition (4.6), almost all Hilbert spaces $H(z)$ are infinite-dimensional. Finally, $\Gamma$ is not amenable.

8.3. An example with an amenable, non-residually finite group. Kirchberg mentioned in [Kir94, Section 5] an interesting example of a finitely generated amenable group which is not residually finite: Denote by $S_{0}$ the group of permutations of $\mathbb{Z}$ which leave unpermuted all but a finite number of integers. We call $A_{0}$ the normal subgroup of even permutations in $S_{0}$. Let $\mathbb{Z}$ act on $S_{0}$ as shift operator. Then the semi-direct product $\Gamma:=\mathbb{Z} \ltimes S_{0}$ is (finitely) generated by the shift $n \mapsto n+1$ and the transposition interchanging 0 and 1 . Note that $\Gamma$ and $S_{0}$ are ICC groups. 
Lemma 8.6. The group $\Gamma$ is amenable. Moreover, $\mathfrak{R}_{\mathcal{F}}(\Gamma)=A_{0}$, hence $\Gamma$ is not residually finite.

Proof. The group $S_{0}$ is amenable as inductive limit of amenable groups; therefore, $\Gamma$ is amenable as semi-direct product of amenable groups (cf. [Day57, Section 4]).

The equality $\mathfrak{R}_{\mathcal{F}}(\Gamma)=A_{0}$ follows from the fact that $A_{0}$ is simple.

Proposition 8.7. Every finite-dimensional unitary representation $\rho$ of $\Gamma$ leaves $A_{0}$ elementwise invariant, i.e., $\rho(\gamma)=\mathbb{1}$ for all $\gamma \in A_{0}$.

Proof. Let $\mathcal{E}$ be the class of countable subgroups of $\mathrm{U}(n), n \in \mathbb{N}$, and $\mathcal{F} \mathcal{G}$ the class of finitely generated groups. Note that $\mathcal{F} \subset \mathcal{E} \cap \mathcal{F G}$ and that finitely generated linear groups are residually finite (cf. Example 6.3 (iii)), i.e. $\mathcal{E} \cap \mathcal{F} \mathcal{G} \subset \mathfrak{R} \mathcal{F}$. Arguing as in the proof of Lemma 8.2 we obtain from the inclusions $\mathcal{F} \subset \mathcal{E} \cap \mathcal{F G} \subset$ $\mathfrak{R F}$ that $\mathfrak{R}_{\mathcal{E} \cap \mathcal{F G}}(\Gamma)=\mathfrak{R}_{\mathcal{F}}(\Gamma)$. Now by Lemma 8.6 the $\mathcal{F}$-residual of $\Gamma$ is $A_{0}$. Finally, since $\Gamma$ itself is finitely generated (i.e. $\Gamma \in \mathcal{F} \mathcal{G}$ ), we have

$$
\mathfrak{R}_{\mathcal{E}}(\Gamma)=\mathfrak{R}_{\mathcal{E} \cap \mathcal{F G}}(\Gamma)=A_{0} .
$$

This concludes the proof since $\rho$ is a finite-dimensional unitary representation iff $\operatorname{im}(\rho) \cong \Gamma / \operatorname{ker} \rho \in \mathcal{E}$, i.e., $\mathfrak{R}_{\mathcal{E}}(\Gamma)$ is the intersection of all ker $\rho$, where $\rho$ are the finite-dimensional, unitary representations of $\Gamma$.

In conclusion, we cannot analyse the spectrum of $\Delta_{X}$ by none of the above methods since $\Gamma$ is not residually finite (and therefore neither of type I). Nevertheless, equality holds in (4.10), but we would need infinite-dimensional Hilbert spaces $H(z)$ in the direct integral decomposition in order to describe the spectrum of the whole covering $X \rightarrow M$ and not only of the subcovering $X / A_{0} \rightarrow M$ (with covering group $\mathbb{Z} \times \mathbb{Z}_{2}$, cf. Diagram (6.1))

\section{OUTLOOK}

Coverings with transformation groups as in Subsection 8.3 cannot be treated with the methods developped in this paper. It seems though reasonable that even for non-residually finite groups the construction specified in Section 3 still produces at least $n$ spectral gaps, $n \in \mathbb{N}$. Nevertheless one probably needs to replace the techniques of Section 4 that use the min-max principle in order to prove the existence of spectral gaps for these types of covering manifolds.

A further interesting line of research would be to consider the opposite situation as in the present paper; that means the use of geometry to prevent the appearance of spectral gaps (cf. [Fri91, Maz91]).

\section{ACKNOWLEDGEMENTS}

It is a pleasure to thank Mohamed Barakat for helpful discussions on residually finite groups. We are also grateful to David Krejčiřík and Norbert Peyerimhoff for useful comments. Finally, we would like to thank Volker Enß for valuable remarks and suggestions on the manuscript. 


\section{REFERENCES}

[Ada95] T. Adachi, On the spectrum of periodic Schrödinger operators and a tower of coverings, Bull. London Math. Soc. 27 (1995), 173-176.

[Alp87] R. C. Alperin, An elementary account of Selberg's lemma, Enseign. Math. (2) 33 (1987), 269-273.

[ASS94] T. Adachi, T. Sunada, and P. W. Sy, On the regular representation of a group applied to the spectrum of a tower, Analyse algébrique des perturbations singulières, II (Marseille-Luminy, 1991), Travaux en Cours, vol. 48, Hermann, Paris, 1994, pp. 125133.

[Ati76] M. F. Atiyah, Elliptic operators, discrete groups and von Neumann algebras, Asterisque 32-33 (1976), 43-72.

[BEG03] J. Brüning, P. Exner, and V. A. Geyler, Large gaps in point-coupled periodic systems of manifolds, J. Phys. A 36 (2003), 4875-4890.

[BJR99] O. Bratteli, P. E. T. Jørgensen, and D. W. Robinson, Spectral asymptotics of periodic elliptic operators, Math. Z. 232 (1999), 621-650.

[Bro81] R. Brooks, The fundamental group and the spectrum of the Laplacian, Comment. Math. Helv. 56 (1981), 581-598.

[Bro86] Robert Brooks, The spectral geometry of a tower of coverings, J. Differential Geom. 23 (1986), 97-107.

[BS92] J. Brüning and T. Sunada, On the spectrum of periodic elliptic operators, Nagoya Math. J. 126 (1992), 159-171.

[CF81] I. Chavel and E. A. Feldman, Spectra of manifolds with small handles, Comment. Math. Helv. 56 (1981), 83-102.

[Dav96] E. B. Davies, Spectral theory and differential operators, Cambridge University Press, Cambridge, 1996.

[Day57] M. M. Day, Amenable semigroups, Illinois J. Math. (1957), 509-544.

[Dix81] J. Dixmier, Von Neumann algebras, North-Holland Mathematical Library, vol. 27, North-Holland Publishing Co., Amsterdam, 1981.

[EP05] P. Exner and O. Post, Convergence of spectra of graph-like thin manifolds, to appear in Journal of Geometry and Physics (2005).

[Fri91] L. Friedlander, Some inequalities between Dirichlet and Neumann eigenvalues, Arch. Rational Mech. Anal. 116 (1991), 153-160.

[Gru01] M. J. Gruber, Noncommutative Bloch theory, J. Math. Phys. 42 (2001), 2438-2465.

[HP03] R. Hempel and O. Post, Spectral gaps for periodic elliptic operators with high contrast: An overview, Progress in Analysis, Proceedings of the 3rd International ISAAC Congress Berlin 20011 (2003), 577-587.

[HR70] E. Hewitt and K. A. Ross, Abstract harmonic analysis. Vol. II, Springer-Verlag, New York, 1970.

[Kal70] R. R. Kallman, A theorem on discrete groups and some consequences of Kazdan's thesis., J. Functional Analysis 6 (1970), 203-207.

[Kan68] E. Kaniuth, Über Charaktere semi-direkter Produkte diskreter Gruppen, Math. Z. 104 (1968), 372-387.

[Kan69] — Der Typ der regulären Darstellung diskreter Gruppen, Math. Ann. 182 (1969), 334-339.

[Kat95] T. Kato, Perturbation theory for linear operators, Classics in Mathematics, SpringerVerlag, Berlin, 1995.

[Kir94] E. Kirchberg, Discrete groups with Kazhdan's property T and factorization property are residually finite, Math. Ann. 299 (1994), 551-563. 
[KK03] D. Krejčiřík and H. Křiž, On the spectrum of curved quantum waveguides, arXiv:math$\mathrm{ph} / 0306008$ (2003).

[KOS89] T. Kobayashi, K. Ono, and T. Sunada, Periodic Schrödinger operators on a manifold, Forum Math. 1 (1989), 69-79.

[LP04] F. Lledó and O. Post, Generating spectral gaps by geometry, arXiv:math-ph/0406032 (2004).

[Mac76] G. W. Mackey, The theory of unitary group representations, University of Chicago Press, Chicago, 1976.

[Mag69] W. Magnus, Residually finite groups, Bull. Amer. Math. Soc. 75 (1969), 305-316.

[Maz91] R. Mazzeo, Remarks on a paper of L. Friedlander concerning inequalities between Neumann and Dirichlet eigenvalues, Internat. Math. Res. Notices (1991), 41-48.

[MM99] M. Marcolli and V. Mathai, Twisted index theory on good orbifolds I: Noncommutative Bloch theory, Commun. Contemp. Math. 1 (1999), 553-587.

[Moo72] C. C. Moore, Groups with finite dimensional irreducible representations, Trans. Amer. Math. Soc. 166 (1972), 401-410.

[Pos03] O. Post, Periodic manifolds with spectral gaps, J. Diff. Equations 187 (2003), 23-45.

[Pyt79] T. Pytlik, A Plancherel measure for the discrete Heisenberg group, Colloq. Math. 42 (1979), 355-359.

[Rat94] J. G. Ratcliffe, Foundations of hyperbolic manifolds, Graduate Texts in Mathematics, vol. 149, Springer-Verlag, New York, 1994.

[Rob82] D. J. S. Robinson, A course in the theory of groups, Graduate Texts in Mathematics, vol. 80, Springer-Verlag, New York, 1982.

[Rob83] A. Robert, Introduction to the representation theory of compact and locally compact groups, London Mathematical Society Lecture Note Series, vol. 80, Cambridge University Press, Cambridge, 1983.

[RS80] M. Reed and B. Simon, Methods of modern mathematical physics I: Functional analysis, Academic Press, New York, 1980.

[Sun88] T. Sunada, Fundamental groups and Laplacians, Geometry and analysis on manifolds, Lecture Notes Mathematics 1339, Springer, Berlin, 1988, pp. 248-277.

[Sun90] , A periodic Schrödinger operator on an abelian cover, J. Fac. Sci. Univ. Tokyo Sect. IA Math. 37 (1990), 575-583.

[Sun92] - Group $C^{*}$-algebras and the spectrum of a periodic Schrödinger operator on a manifold, Can. J. Math. 44 (1992), 180-193.

[Tho64] E. Thoma, Über unitäre Darstellungen abzählbarer, diskreter Gruppen, Math. Ann. 153 (1964), 111-138.

[Tho68] - Eine Charakterisierung diskreter Gruppen vom Typ I, Invent. Math. 6 (1968), 190-196.

[Uhl76] K. Uhlenbeck, Generic properties of eigenfunctions, Amer. J. Math. 98 (1976), 10591078.

[Wal92] N. R. Wallach, Real reductive groups. II, Pure and Applied Mathematics, vol. 132, Academic Press, Boston, 1992.

Institut für Reine und Angewandte Mathematik, Rheinisch-Westfälische TeChnische Hochschule Aachen, Templergraben 55, D-52062 Aachen, Germany

E-mail address: lledo@iram.rwth-aachen.de

E-mail address: post@iram.rwth-aachen.de 